\title{
CATEGORICAL COMPLEXITY
}

\author{
SAUGATA BASU ${ }^{\circledR 1}$ and UMUT ISIK ${ }^{2}$ \\ ${ }^{1}$ Department of Mathematics, Purdue University, West Lafayette, IN 47906, USA; \\ email: sbasu@math.purdue.edu \\ 2 Department of Mathematics, University of California, Irvine, Irvine, CA 92697, USA; \\ email: isik@math.uci.edu
}

Received 14 January 2018; accepted 21 March 2020

\begin{abstract}
We introduce a notion of complexity of diagrams (and, in particular, of objects and morphisms) in an arbitrary category, as well as a notion of complexity of functors between categories equipped with complexity functions. We discuss several examples of this new definition in categories of wide common interest such as finite sets, Boolean functions, topological spaces, vector spaces, semilinear and semialgebraic sets, graded algebras, affine and projective varieties and schemes, and modules over polynomial rings. We show that on one hand categorical complexity recovers in several settings classical notions of nonuniform computational complexity (such as circuit complexity), while on the other hand it has features that make it mathematically more natural. We also postulate that studying functor complexity is the categorical analog of classical questions in complexity theory about separating different complexity classes.
\end{abstract}

2010 Mathematics Subject Classification: 18A10, 68Q15 (primary); 14Q20 (secondary)

\section{Introduction}

It is usual to associate some measure of complexity to mathematical objects. For example, the complexity of a polynomial is often measured by its degree, or alternatively by the volume of its Newton polytope, or the number the monomials appearing in it with nonzero coefficients, or the least number of operations needed for an algorithm to evaluate the polynomial at a given point. Once a notion of complexity is fixed, one can make quantitative statements about properties of the objects in terms of their complexity. In the case of polynomials for example, there are many results on upper bounds on the topological invariants of the variety that

(C) The Author(s) 2020. This is an Open Access article, distributed under the terms of the Creative Commons Attribution-NonCommercialNoDerivatives licence (http://creativecommons.org/licenses/by-nc-nd/4.0/), which permits non-commercial re-use, distribution, and reproduction in any medium, provided the original work is unaltered and is properly cited. The written permission of Cambridge University Press must be obtained for commercial re-use or in order to create a derivative work. 
the polynomial defines (see for example, the survey [BPR05]), the number of steps needed to desingularize the variety [BGMW11], and many other functions defined on the space of polynomials, in terms of the chosen complexity measure.

The notion of complexity also arose in theoretical computer science as a means of studying efficiency of algorithms and also to measure the intrinsic hardness of certain algorithmic problems. The latter led to the development of structural complexity theory and in particular to the famous $\mathrm{P}$ versus NP questions for discrete complexity classes, which remain unresolved until today. Even though these arose first in the context of decision problems and Boolean functions, there have been subsequent attempts to generalize the scope of computational complexity to other classes of mathematical objects - for example, the BlumShub-Smale (B-S-S) theory for computations over reals as well as complex numbers [BCSS98], over more general structures [Poi95], for polynomials [Val79a, Val79b, vzG87], and for constructible sheaves and functions [Bas15]. Some of these generalizations are motivated by costs of computations in certain models of computations, while others by the desire to have a sound internal notion of complexity for mathematical objects. Remarkably, there exist analogs of the P versus NP question in all the generalizations mentioned above. Thus, it seems that there should be a more fundamental way of looking at questions arising in computational complexity theory, which unifies these various viewpoints.

The goal of the current paper is to develop this general theory of complexity via a categorical approach that reconciles the intuitive notion of complexity of mathematical objects with the different notions of computational and circuit complexities used in theoretical computer science.

We start by defining a categorical notion called a diagram computation. In an arbitrary category $\mathcal{C}$ with a chosen set of morphisms called basic morphisms, diagram computations can be used to construct diagrams and, in particular, objects and morphisms in $\mathcal{C}$ as follows. At the first level, one starts with a diagram consisting entirely of basic morphisms and then successively adds limits and colimits of arbitrary subdiagrams, along with the accompanying morphisms from/to those subdiagrams, to construct more and more complex diagrams. Any diagram isomorphic to a subdiagram of the final resulting diagram is said to be computed by the diagram computation. This allows us to associate, to each object, arrow, or diagram in $\mathcal{C}$, a complexity by counting the number of limits, colimits, and basic morphisms used in its most efficient computation. Diagram computations come in three kinds, the full version described above, called a mixed computation, and two more restricted ones where one is either allowed to use only the so-called constructive limits or only constructive colimits. This leads to the notions of mixed complexity, limit complexity, and colimit complexity of objects, arrows, or any diagrams in $\mathcal{C}$. 
Although our notion of complexity bears some similarity with a more classical view of complexity coming from logic, namely descriptive complexity [Imm95], there is one important respect in which our notion of complexity differs significantly from all classical notions. In our categorical world, isomorphic objects should have identical complexity-which is indeed the case with our definition. Thus, we are able to define a good notion of complexity in the category, say, of affine or projective schemes, which is independent of embeddings. This is very natural from the mathematical point of view-making our theory completely geometric in those settings - but is sometimes at odds with ordinary complexity theory, which deals with embedded objects (like subsets of the Boolean hypercube or subvarieties of $\mathbb{C}^{n}$ ). Nevertheless, we will show that, with the appropriate choice of category and basic morphisms, even noncategorical notions of complexity can be meaningfully embedded in categorical ones.

A fundamentally new point of view emerges when one thinks about the categorical analogs of classical complexity questions. For every functor between two categories for which complexity is defined, one can define a natural notion of complexity of the functor. Unlike, the complexity of diagrams, which are numbers, the complexity of a functor is a function $f: \mathbb{N} \rightarrow \mathbb{N}$, and one can ask whether the complexity of such a functor is bounded from above by a polynomial. In this way, classical questions about separation of complexity classes become, in the categorical world, questions about polynomial boundedness of the complexity of certain natural functors. With this shift of viewpoint, one can pose many questions about complexities of functors, which have no direct analogs in the world of computational complexity. Well-studied properties of functors such as preservation of limits and colimits, adjointness, and so on are important from this point of view.

The importance of functor complexity was already suggested in [Bas15], where the complexities of adjoint pairs of functors between the categories of semialgebraically constructible sheaves on finite-dimensional real affine spaces were posited as generalizing the $\mathrm{P}$ versus NP question in the real $\mathrm{B}-\mathrm{S}-\mathrm{S}$ model.

We remark here that connections between computability and logic on one hand and category theory and topos theory on the other hand have a long history (see for example, [LS88, MLM94]). A more recent work on computability and complexity in categorical structures using the notion of Kan extensions appears in [Yan15]. However, our goal is different, and it is to develop a completely general notion of complexity, based on category theory, that is useful in studying basic objects in algebra and geometry from a quantitative point of view. To the best of our knowledge, this task has not been undertaken before.

We now give a brief summary of our results. After the basic definitions in Section 3, we look at several key examples. For sets, the colimit complexity of 
a set $S$ is card $(S)+1$ (Proposition 3.15). Infinite sets are 'noncomputable' in this theory. For topological spaces, colimit computations starting from simplices and face maps define a simplicial complexity for topological spaces. Similarly, mixed computations starting from points and intervals give rise to cubical complexity. These measure how hard it is to make a space from simplices and cubes, respectively. Another important example is one where we recover monotone Boolean complexity from the categorical complexity in the lattice of subsets of a finite set.

In order to relate the new notion of categorical complexity, with pre-existing notions of (nonuniform) complexity, such as circuit complexity, or lengths of straight-line programs (we refer the reader to the books [BCS97, Bür00] for these notions), we prove certain comparison theorems. The first set of such theorems is about affine varieties, affine schemes, and algebras over a field. We show that the affine zero-set of a polynomial with low arithmetic circuit complexity has low limit complexity (Theorem 4.2); on the other hand, if $X$ is a variety with low limit complexity, then it is isomorphic to the zero-set of a polynomial with low arithmetic circuit complexity (Theorem 4.5). The same results hold for affine schemes and algebras. For projective schemes in $\mathbb{P}^{n}$ : by building affine pieces with limits and then gluing them using colimits, we show that the mixed complexity of a projective scheme is bounded above by a constant multiple of $n^{2} N$, where $N$ is the arithmetic circuit complexity of its defining equations.

The categorical complexities of isomorphic varieties are equal by definition, while circuit complexity, being a nongeometric attribute, does not share this property. In order to reconcile these two notions, we consider in Section 5 two additional categories where circuit complexity of polynomials is, in a sense, embedded into categorical complexity. The first of these is the category of pairs of graded algebras, constructed specifically to make this embedding possible. Still, it remains to be seen how complexity in this category compares to arithmetic circuit complexity of polynomials, or to the complexity of projective varieties discussed in [Isi19]. The second category considered here is the category of modules over polynomial rings, where we prove that the colimit complexities of a sequence of morphism diagram $\left(k\left[x_{1}, \ldots, x_{n}\right] \stackrel{1 \mapsto f_{n}}{\longrightarrow} k\left[x_{1}, \ldots, x_{n}\right]\right)_{n>0}$ are bounded by a quasipolynomial function of $n$, if and only if the arithmetic circuit complexities of the sequence $\left(f_{n}\right)_{n>0}$ are also bounded by a quasipolynomial function of $n$ (cf. Remark 5.8).

In Section 6, we discuss the behavior of categorical complexity under the action of functors. Limit and colimit computations are preserved under right and left adjoints, respectively. We define the complexity of a functor $F: \mathcal{C} \rightarrow \mathcal{D}$ as a function $C(F)(n)$ of $n$, where $C(F)(n)$ is the supremum of the complexity of $F(D)$, where $D$ runs over all diagrams in $\mathcal{C}$ whose complexity is less than or 
equal to $n$. We posit that the question of whether the complexity of the 'image functor' (see Definition 6.8) on the morphism category $\mathcal{C}^{\bullet} \rightarrow \cdot$ is polynomially bounded, is the categorical analog of the $\mathrm{P}$ versus NP problem for the category $\mathcal{C}$ (cf. discussion in the beginning of Section 6.4). We investigate this question in the context of limit complexity in the categories of semilinear and semialgebraic sets and answer it in the negative. Our final result is an analysis of the colimit complexity of the image functor for the category of modules over polynomial rings.

Finally, in Section 7, we list several open problems and future research direction in the area of categorical complexity.

We assume no prior knowledge of category theory in this paper and have included all relevant definitions. For background in category theory, we refer the reader to the books [Awo10, ML98, Sim11]. For background in complexity theory, we refer the reader to the books [BCS97, Bür00]. Finally, we make use of certain basic functors from algebraic geometry, and we refer the reader to the book [MO15] as an accessible source for these.

\section{Categories and functors}

In this section, we recall some basic definitions from category theory and introduce some notation that will be useful in what follows.

Definition 2.1 (Categories). A category $\mathcal{C}$ consists of

(1) a class $\operatorname{Ob}(\mathcal{C})$ whose elements are the 'objects of the category $\mathcal{C}^{\prime}$;

(2) for every pair $A, B$ of objects of $\mathcal{C}$, a $\operatorname{set} \mathcal{C}(A, B)$ of 'morphisms' or 'arrows' from $A$ to $B$;

(3) for every triple $A, B, C$ of objects of $\mathcal{C}$, a composition law

$$
\mathcal{C}(A, B) \times \mathcal{C}(B, C) \rightarrow \mathcal{C}(A, C),
$$

which will be denoted by $(f, g) \mapsto g \circ f$;

(4) for every object $A$ of $\mathcal{C}$, a morphism $1_{A} \in \mathcal{C}(A, A)$ called the identity morphism on $A$.

The above data are subject to the following two axioms.

(a) (Associativity). Given morphisms $f \in \mathcal{C}(A, B), g \in \mathcal{C}(B, C), h \in \mathcal{C}(C, D)$,

$$
h \circ(g \circ f)=(h \circ g) \circ f .
$$


(b) (Identity). Given morphisms $f \in \mathcal{C}(A, B), g \in \mathcal{C}(B, C)$, the following equalities hold.

$$
\begin{aligned}
& 1_{B} \circ f=f, \\
& g \circ 1_{B}=g .
\end{aligned}
$$

We say that a category $\mathcal{C}$ is a small category if its class of objects is a set.

For any category $\mathcal{C}$, we will denote by $\mathcal{C}^{\text {opp }}$ the category whose morphisms are defined by

$$
\mathcal{C}^{\text {opp }}(A, B)=\mathcal{C}(B, A)
$$

for every pair of objects $A, B$ of $\mathcal{C}$.

Notation 2.2. We will denote a morphism $f \in \mathcal{C}(A, B)$ often as $f: A \rightarrow B$, and also denote the source $A$ by $\operatorname{dom}(f)$, and the target $B$ by $\operatorname{codom}(f)$.

NotATION 2.3. The following categories will appear later in the paper.

(A) The category Set, whose objects are sets and whose morphisms are maps between sets.

(B) The category Vect $_{k}$, where $k$ is a field, and whose objects are $k$-vector spaces and whose morphisms are linear maps.

(C) The category Grp of groups and homomorphisms.

(D) The category SL (respectively, SA) of embedded semilinear (respectively, semialgebraic) sets and affine (respectively, polynomial) maps. More precisely, each object of SL (respectively, SA) is a semilinear (respectively, semialgebraic) subset $A \subset \mathbb{R}^{n}$ for some $n \geqslant 0$, and a morphism $\left(A \subset \mathbb{R}^{n}\right) \rightarrow\left(B \subset \mathbb{R}^{m}\right)$ is a map $f: A \rightarrow B$ such that there exists a commutative square

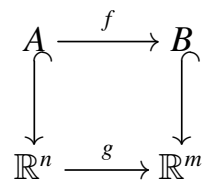

where $g: \mathbb{R}^{n} \rightarrow \mathbb{R}^{m}$ is an affine (respectively, polynomial) map. In other words, $f$ is the restriction to $A$ of an affine (respectively, polynomial) map from $\mathbb{R}^{n}$ to $\mathbb{R}^{m}$.

(E) The category $\mathbf{A l g}_{k}$ of $k$-algebras over a field $k$. 
(F) The category $\mathbf{A f f V a r}_{k}$ of affine varieties and the category $\mathbf{A f S S c h}_{k}$ of affine $k$-schemes for a field $k$.

(G) The category $R$-Mod, where $R$ is a polynomial ring in finitely many variables.

(H) The category Top of topological spaces.

Definition 2.4 (Functors). A (covariant) functor $F$ from a category $\mathcal{A}$ to $\mathcal{B}$ consists of the following:

(1) a mapping $\mathrm{Ob}(\mathcal{A}) \rightarrow \mathrm{Ob}(\mathcal{B})$ (the image of $A$ will be written as $F(A)$ );

(2) for every pair of objects $A, A^{\prime}$ of $\mathcal{A}$, a mapping $\mathcal{A}\left(A, A^{\prime}\right) \rightarrow \mathcal{B}(F(A)$, $F\left(A^{\prime}\right)$ ) (the image of $f \in \mathcal{A}\left(A, A^{\prime}\right)$ ) is written as $F(f)$ ).

The above data is subject to the following axioms.

(a) for every pair of morphisms $f \in \mathcal{A}\left(A, A^{\prime}\right), g \in \mathcal{A}\left(A^{\prime}, A^{\prime \prime}\right)$,

$$
F(g \circ f)=F(g) \circ F(f)
$$

(b) for every object $A$ of $\mathcal{A}$,

$$
F\left(1_{A}\right)=1_{F(A)}
$$

A contravariant functor $F$ from a category $\mathcal{A}$ to $\mathcal{B}$ is a covariant functor from $\mathcal{A}^{\text {opp }}$ to $\mathcal{B}$.

DEFINITION 2.5 (Natural transformations between functors). Let $F, G$ be two (covariant) functors from a category $\mathcal{C}$ to $\mathcal{D}$. A natural transformation $\theta: F \rightarrow G$ is a family of arrows $\left(\theta_{C}: F(C) \rightarrow G(C)\right)_{C}$ in $\mathcal{D}$, indexed by objects $C$ of $\mathcal{C}$, such that for each pair of objects $C, C^{\prime}$ of $\mathcal{C}$ and $f \in \mathcal{C}\left(C, C^{\prime}\right)$, the following diagram commutes:

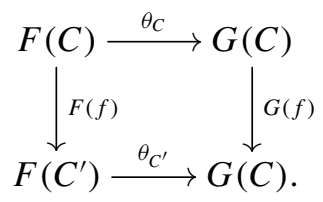

We will denote the class of all natural transformations between two functors $F, G$ by $\operatorname{Nat}(F, G)$. A natural transformation $\theta \in \operatorname{Nat}(F, G)$ is called an isomorphism if it admits an inverse. 
Definition 2.6. Two functors $F: \mathcal{C} \rightarrow \mathcal{D}$ and $U: \mathcal{D} \rightarrow \mathcal{C}$ are said to be an adjoint pair (with $F$ left-adjoint to $U$ and $U$ right-adjoint to $F$ ), if there exist for each object $C$ of $\mathcal{C}$ and $D$ of $\mathcal{D}$, bijective maps

$$
\begin{aligned}
& \mathcal{C}(C, U(D)) \rightarrow \mathcal{D}(F(C), D), f \mapsto f^{\sharp}, \\
& \mathcal{D}(F(C), D) \rightarrow \mathcal{C}(C, U(D)), \quad g \mapsto g_{\mathrm{b}},
\end{aligned}
$$

which are inverses to each other and are moreover natural in $C$ and $D$.

More precisely, for every $k \in \mathcal{C}(A, C), \ell \in \mathcal{D}(D, B)$,

$$
\begin{gathered}
(U(\ell) \circ f \circ k)^{\sharp}=\ell \circ f^{\sharp} \circ F(k), \\
U(\ell) \circ g_{b} \circ k=(\ell \circ g \circ F(k))_{b}
\end{gathered}
$$

both hold.

Remark 2.7. In Definition 2.6, the functors $F, U$ induce functors $\mathcal{F}, \mathcal{U}$ from the product category $\mathcal{C}^{\mathrm{opp}} \times \mathcal{D}$ to Set defined by

$$
\begin{aligned}
& \mathcal{F}(C, D)=\mathcal{D}(F(C), D), \\
& \mathcal{U}(C, D)=\mathcal{C}(C, U(D)),
\end{aligned}
$$

with $\mathcal{F}(f, g), \mathcal{G}(f, g)$ defined in the obvious manner for $f, g$ arrows in the categories $\mathcal{C}^{\text {opp }}$ and $\mathcal{D}$, respectively. Then, the naturality condition in Definition 2.6 translates into the fact that the functors $\mathcal{F}$ and $\mathcal{U}$ are natural transformations.

EXAMPLE 2.8. The functor $F:$ Set $\rightarrow$ Grp, which takes a set to the free group generated by the set, and the forgetful functor $U:$ Grp $\rightarrow$ Set form an adjoint pair (with $F$ left-adjoint to $U$ ).

We also need the notion of universal elements.

DEFINITION 2.9 (Representable functors, Yoneda's lemma, and universal elements). Let $\mathcal{C}$ be a category and $F$ a functor from $\mathcal{C}$ to Set. Then for each object $A$ of $\mathcal{C}, \mathcal{C}(A, \cdot)$ is a functor from $\mathcal{C}$ to Set. The map

$$
\lambda_{A}: \operatorname{Nat}(\mathcal{C}(A, \cdot), F) \rightarrow F(A)
$$

defined by

$$
\lambda_{A}(\phi)=\phi_{A}\left(\mathrm{id}_{A}\right)
$$


is bijective. This statement is referred to as Yoneda's lemma. Now, if $\phi \in$ $\operatorname{Nat}(\mathcal{C}(A, \cdot), F)$ is an isomorphism, then we say that $F$ is representable (by $A$ ), and $u=\lambda_{A}(\phi)$ is called a universal element of $F$. The element $u$ has the property, that for any object $B$ of $\mathcal{C}$ and $f \in \mathcal{C}(B, A)$, there is a unique element $t \in F(B)$, such that $u=F(f)(t)$.

If $F$ is a contravariant functor from $\mathcal{C}$ to $(\mathrm{Set})$, then it is representable by an object $A$ of $\mathcal{C}$, if and only if the corresponding covariant functor from $\mathcal{C}^{\text {opp }}$ to sets is representable, and in this case a universal element of this covariant functor will be called a universal element of $F$.

DEFINITION 2.10 (Graphs and diagram categories). A directed graph $I$ is a quadruple $(V, E, s, t)$, where $V, E$ are sets (referred to as the sets of vertices and edges of $I$ ) and $s, t: E \rightarrow V$ are maps. (For $e \in E$, we will sometimes refer to $s(e)$ as the source and $t(e)$ as the target of the edge $e$.) A homomorphism $\phi=\left(\phi_{1}, \phi_{2}\right): I \rightarrow I^{\prime}$ of directed graphs $I=(V, E, s, t), I^{\prime}=\left(V^{\prime}, E^{\prime}, s^{\prime}, t^{\prime}\right)$ is a pair of maps $\phi_{1}: V \rightarrow V^{\prime}, \phi_{2}: E \rightarrow E^{\prime}$ such that the two diagrams

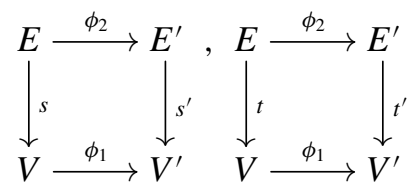

commute.

For a small category $\mathcal{C}$, we will denote by $U(\mathcal{C})$ the directed graph whose set of vertices is the set of objects of $\mathcal{C}$ and whose set of edges is the set of all morphisms of $\mathcal{C}$, along with the maps $s, t$ taking a morphism to its codomain and domain, respectively. Even if the category $\mathcal{C}$ is not small, we will continue to use the notation $U(\mathcal{C})$ to denote its underlying graph, keeping in mind that this will always restrict our attention to subgraphs of $U(\mathcal{C})$ with finite sets of vertices.

Let $\mathcal{C}$ be a category and let $U(\mathcal{C})$ be the underlying directed graph. Let $I=(V$, $E, s, t)$ be any directed graph. By a diagram in $\mathcal{C}$, we mean a directed graph homomorphism $D: I \rightarrow U(\mathcal{C})$. The graph $I$ will be called the shape of $D$, and we will denote by $\mathrm{v}(D)$ (respectively, e $(D), s(D), t(D)$ ) the set of vertices (respectively, edges, sources, and targets of edges) of the graph $I$.

We say that the diagram $D$ is discrete if the edge set $E$ of $I$ is empty.

By a subdiagram of a diagram $D: I \rightarrow U(\mathcal{C})$, with $I=(V, E)$, we mean the restriction $D_{J}: J \rightarrow U(\mathcal{C})$, with $J=\left(V^{\prime}, E^{\prime}\right)$ a full subgraph of $I$, that is, $V^{\prime} \subset V$, and $E^{\prime}=\left\{e \in E \mid s(e) \in V^{\prime}, t(e) \in V^{\prime}\right\}$. The restrictions to not necessarily full subgraphs will be specified as not necessarily full subdiagrams. 
For $I=(V, E, s, t)$ and two diagrams, $D_{1}: I \rightarrow U(\mathcal{C}), D_{2}: I \rightarrow U(\mathcal{C})$, a morphism between $D_{1}$ and $D_{2}$ is a collection of morphisms $\varphi=\left(\varphi_{v}: D_{1}(v) \rightarrow\right.$ $\left.D_{2}(v)\right)_{v \in V}$ such that for all $e \in E, D_{2}(e) \circ \varphi_{D_{1}(s(e))}=\varphi_{D_{1}(t(e))} \circ D_{1}(e)$. This defines the category of diagrams, $\mathcal{C}^{I}$, of the category $\mathcal{C}$ with shape $I$.

Remark 2.11. Note that in Definition 2.10, $I$ is just a directed graph and does not have a composition operation on it. As such, there is no a priori assumption of functoriality/commutativity for diagrams.

It is possible to associate a category, $\mathbf{P t h}(I)$, to each directed graph $I$, called the path category of $I$, and define (path) diagrams as actual functors from $\operatorname{Pth}(I)$ to a category $\mathcal{C}$ [Sim11]. The diagrams we consider in this paper are not functors in this sense.

Also, note that our notion of a full subdiagram is not the same as the full subfunctor of the diagram functor from the path category of $I$. So if $I$ is the directed graph $1 \rightarrow 2 \rightarrow 3$ and $D$ a diagram of a category $\mathcal{C}$ of shape $I$, then the restriction of $D$ to the full subgraph corresponding to the vertex set $J=\{1,3\}$ is the diagram whose image is the subgraph of $U(\mathcal{C})$ consisting of two vertices $D(1), D(3)$ and an empty set of edges.

DEFINITION 2.12 (Cones and limits). Given an object $W$ of $\mathcal{C}$, a constant diagram with value $W$ and shape $I=(V, E, s, t)$ is the diagram $D: I \rightarrow U(\mathcal{C})$, with $D(v)=W$ for all $v \in V$ and $D(e)=1_{W}$ for all $e \in E$.

Given a diagram $D: I \rightarrow U(\mathcal{C})$ and an object $W$ of $\mathcal{C}$, a cone over $D$ with vertex $W$ is a morphism $D^{\prime} \rightarrow D$, where $D^{\prime}$ is a constant diagram of shape $I$ with value $W$. Note that if $D^{\prime}$ is the constant diagram of shape $I$ and value $W$, then a cone $\varphi: D^{\prime} \rightarrow D$ is determined by morphisms $\varphi(v): W \rightarrow D(v)$ such that the following diagram commutes for each $e \in E$ :

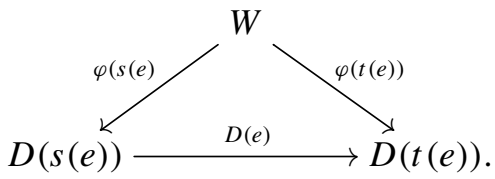

Note that the cone over $D: I \rightarrow \mathcal{C}$ with vertex $W$ can also be thought of as a new diagram, whose associated directed graph is obtained from the graph of $I$ by adding one extra vertex $v_{0}$ and an edge, $e_{v}$, for each vertex $v$ of $I$, with source $v_{0}$ and target $v$ (cf. Figure 1). This diagram of course has the extra property that all subdiagrams of the form (1) commute.

Given a diagram $D: I \rightarrow U(\mathcal{C})$, with $I=(V, E, s, t)$, the map $\mathcal{C}^{I}(\cdot, D)$, that associates to each object $W$ of $\mathcal{C}$ the set of cones over $D$ with vertex $W$ defines a contravariant functor from $\mathcal{C}$ to Set. 


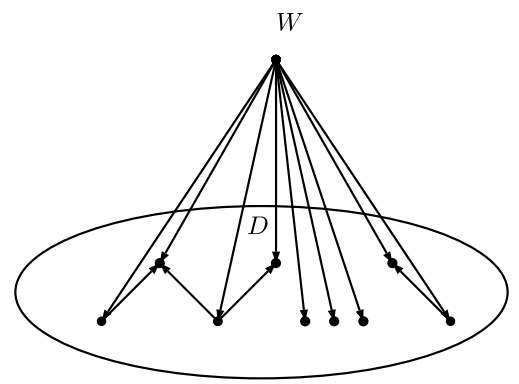

Figure 1. Cone over $D$ with vertex $W$.

A universal element of this functor is called the limit of $D($ denoted by $\lim D)$. In other words, $\lim D$ is a cone $\lim D: D^{\prime} \rightarrow D$, where $D^{\prime}$ is a constant diagram of shape $I$ (say with value $L$ ) such that for any cone $\varphi: D^{\prime \prime} \rightarrow D$, where $D^{\prime \prime}$ is a constant diagram of shape $I$ with value $M$, there exists a unique morphism $\varphi^{\prime}: M \rightarrow L$ such that the following diagram commutes (denoting by $\varphi^{\prime}$ also the induced morphism in $\left.\mathcal{C}^{I}\left(D^{\prime \prime}, D\right)\right)$ :

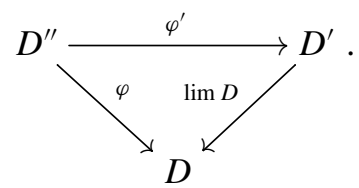

We will denote by $I(\lim D)$ the directed graph $\left(V^{\prime}, E^{\prime}, s^{\prime}, t^{\prime}\right)$, where

$$
\begin{aligned}
& V^{\prime}=V \cup\left\{v_{0}\right\}, \quad v_{0} \notin V, \\
& E^{\prime}=E \cup \bigcup_{v \in V}\left\{e_{v}\right\},
\end{aligned}
$$

and $s^{\prime}, t^{\prime}$ are defined by

$$
\begin{gathered}
\left.\begin{array}{c}
s^{\prime}(e)=s(e), \\
t^{\prime}(e)=t(e),
\end{array}\right\} \quad \text { if } e \in E, \\
\left.\begin{array}{l}
s^{\prime}(e)=v_{0}, \\
t^{\prime}(e)=v .
\end{array}\right\} \quad \text { if } e=e_{v}, v \in V .
\end{gathered}
$$

We will also sometimes abuse notation and refer to the value $L$ of the limit also by $\lim D$. 
EXAMPLE 2.13 (Products, pullbacks, equalizers, and composition). The limit of a discrete diagram is called a product. Limits of diagrams of shapes

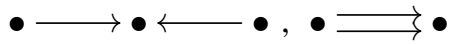

are usually referred to as pullbacks and equalizers, respectively. Note also that taking the composition of two morphisms is a particular case of taking limits of diagrams of shape

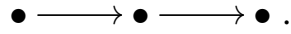

DEFINITION 2.14 (Cocones and colimits). By dualizing (that is, reversing the direction of the arrow) in Definition 2.12, we obtain the notion of cocones and colimits of diagrams. Given a diagram $D: I \rightarrow U(\mathcal{C})$ and an object $W$ of $\mathcal{C}$, a cocone over $D$ with vertex $W$ is a morphism $D \rightarrow D^{\prime}$, where $D^{\prime}$ is a constant diagram of shape $I$ with value $W$. Note that if $D^{\prime}$ is the constant diagram of shape $I$ and value $W$, then a cone $\varphi: D \rightarrow D^{\prime}$ is determined by morphisms $\varphi(v): D(v) \rightarrow W$ such that the following diagram commutes for each $e \in E$ :

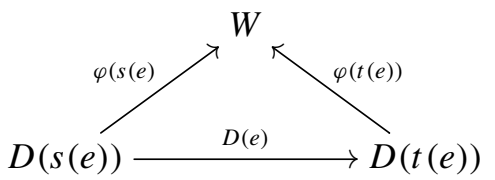

Given a diagram $D: I \rightarrow U(\mathcal{C})$, with $I=(V, E, s, t)$, the map $\mathcal{C}^{I}(D, \cdot)$, that associates to each object $W$ of $\mathcal{C}$ the set of cocones over $D$ with vertex $W$ defines a covariant functor from $\mathcal{C}$ to Set. A universal element of this functor is called the colimit of $D$ (denoted by colim $D$ ). In other words, colim $D$ is a cocone colim $D: D \rightarrow D^{\prime}$, where $D^{\prime}$ is a constant diagram of shape $I$ (say with value $C$ ) such that for any cocone $\varphi: D \rightarrow D^{\prime \prime}$, where $D^{\prime \prime}$ is a constant diagram of shape $I$ with value $M$, there exists a unique morphism $\varphi^{\prime}: C \rightarrow M$ such that the following diagram commutes (denoting by $\varphi^{\prime}$ also the induced morphism in $\mathcal{C}^{I}\left(D^{\prime \prime}, D\right)$ ):

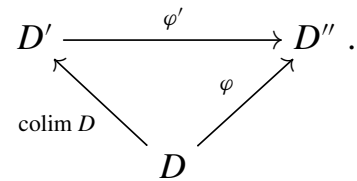

We will denote by $I(\operatorname{colim} D)$ the directed graph $\left(V^{\prime}, E^{\prime}, s^{\prime}, t^{\prime}\right)$, where

$$
\begin{aligned}
& V^{\prime}=V \cup\left\{v_{0}\right\}, \quad v_{0} \notin V, \\
& E^{\prime}=E \cup \bigcup_{v \in V}\left\{e_{v}\right\},
\end{aligned}
$$


and $s^{\prime}, t^{\prime}$ are defined by

$$
\begin{aligned}
& \left.\begin{array}{l}
s^{\prime}(e)=s(e), \\
t^{\prime}(e)=t(e),
\end{array}\right\} \quad \text { if } e \in E \\
& \left.\begin{array}{l}
s^{\prime}(e)=v, \\
t^{\prime}(e)=v_{0} .
\end{array}\right\} \text { if } e=e_{v}, v \in V .
\end{aligned}
$$

We will sometimes abuse notation and refer to the value $C$ of the colimit also by colim $D$.

EXAMPLE 2.15 (Coproducts, pushforwards, and coequalizers). By reversing the direction of the arrows of the diagrams in Example 2.13 and taking colimits, we obtain the definitions of coproducts, pushforwards, and coequalizers (respectively, from the definitions of products, pullbacks, and equalizers).

\section{Definition of diagram computations}

In this section, we define diagram computations and categorical complexity. As explained in Section 1 (Introduction), diagram computations come in three different flavors-namely, limit, colimit, and mixed limit-colimit computations. The associated notions of complexities of diagrams will be called limit, colimit, and mixed complexity, respectively.

We fix a category $\mathcal{C}$ for the rest of this section and also fix a set $\mathcal{A}$ of morphisms in $\mathcal{C}$. The morphisms in $\mathcal{A}$ will be referred to as the basic morphisms in $\mathcal{C}$.

3.1. Limit and colimit computations. We define a notion of computation in $\mathcal{C}$, called a limit computation, by starting with these basic morphisms and adding a finite limit at each step; similarly, in a colimit computation, we build objects by adding colimits of subdiagrams.

DEFINITION 3.1. A limit computation (respectively, a colimit computation) in $\mathcal{C}$ is a finite sequence of diagrams $\left(D_{0}, \ldots, D_{s}\right)$, with $D_{j}: I_{j}=\left(V_{j}, E_{j}, s_{j}\right.$, $\left.t_{j}\right) \rightarrow U(\mathcal{C}), 0 \leqslant j \leqslant s$, where we have the following:

(i) $D_{0}(e) \in \mathcal{A}$ for each edge $e$ of $I_{0}$.

(ii) For each $i=1, \ldots, s, D_{i}$ is obtained from $D_{i-1}$ by adding a limit or colimit cone of a subdiagram. More precisely, there is a subdiagram $\left.D_{i-1}\right|_{J_{i}}$ of $D_{i-1}$ with

$$
J_{i}=\left(V_{i-1}^{\prime}, E_{i-1}^{\prime}, s_{i}^{\prime}=\left.s_{i}\right|_{E_{i-1}^{\prime}}, t_{i}^{\prime}=\left.t_{i}\right|_{E_{i-1}^{\prime}}\right), \quad V_{i-1}^{\prime} \subset V_{i-1}, E_{i-1}^{\prime} \subset E_{i-1}
$$


such that denoting $I\left(\left.\lim D_{i-1}\right|_{J_{i}}\right)=\left(V^{\prime}, E^{\prime}, s^{\prime}, t^{\prime}\right) \quad$ (respectively, $\left.I\left(\left.\operatorname{colim} D_{i-1}\right|_{J_{i}}\right)=\left(V^{\prime}, E^{\prime}, s^{\prime}, t^{\prime}\right)\right)$,

(1) $V_{i}=V_{i-1} \cup\left\{v_{0}\right\}$, where $v_{0}$ is the unique vertex of $V^{\prime} \backslash V_{i-1}$;

(2) $E_{i}=E_{i-1} \cup \bigcup_{v \in V_{i-1}^{\prime}}\left\{e_{v}\right\}$;

(3) for $e \in E_{i}$,

$$
\begin{aligned}
& \left.\begin{array}{l}
s_{i}(e)=s_{i-1}(e), \\
t_{i}(e)=t_{i-1}(e),
\end{array}\right\} \quad \text { for } e \in E_{i-1}, \\
& \left.\begin{array}{l}
s_{i}(e)=v_{0}(\text { respectively, } v), \\
t_{i}(e)=v\left(\text { respectively, } v_{0}\right),
\end{array}\right\} \quad \text { for } e=e_{v}, v \in V_{i-1}^{\prime}
\end{aligned}
$$

(4) for $e \in E_{i}$,

$$
\begin{aligned}
D_{i}(e)= & D_{i-1}(e) \quad \text { for } e \in E_{i-1}, \\
D_{i}(e)= & \left(\left.\lim D_{i-1}\right|_{J_{i}}\right)_{v}\left(\text { respectively, }\left(\operatorname{colim} D_{i-1}||_{J_{i}}\right)_{v}\right), \\
& \text { for } e=e_{v}, v \in V_{i-1}^{\prime},
\end{aligned}
$$

(cf. Definitions 2.10, 2.12, and 2.14).

(iii) (Constructivity) For each $i, 0<i \leqslant s$, if the unique vertex $v_{0} \in V_{i} \backslash V_{i-1}$ belongs to $J_{j}$ for some $j, i<j \leqslant s$, then $J_{i}$ is a subgraph of $J_{j}$. (In other words, if a limit $L_{i}=\left.\lim D_{i-1}\right|_{J_{i}}$ (respectively, colimit $C_{i}$ ) produced in the $i$ th step of the computation is used again in the subdiagram $\left.D_{j-1}\right|_{J_{j}}$ used at the $j$ th step of the computation, then $J_{i}$ is a subgraph of $J_{j}$, that is, the subdiagram that produced $L_{i}$ (respectively, $C_{i}$ ) must be a subdiagram of $\left.D_{j-1}\right|_{J_{j}}$.)

The computation $\left(D_{0}, \ldots, D_{s}\right)$ is said to compute a diagram $D$ if $D$ is isomorphic to a (not necessarily full) subdiagram of $D_{s}$. In particular, an object in $\mathcal{C}$ is computed by $\left(D_{0}, \ldots, D_{s}\right)$ if an object isomorphic to it appears in $D_{s}$.

REMARK 3.2. Note that in Definition 3.1, we are not assuming that all limits or colimits of finite diagrams exist but in each particular computation $\left(D_{0}, \ldots, D_{s}\right)$, for each $i, 0 \leqslant i<s$, the limit or colimit of the subdiagram $\left.D_{i}\right|_{J_{i}}$ of $D_{i}$ is assumed to exist. Thus, the notion of a limit/colimit computation is still well defined even if the category $\mathcal{C}$ does not admit limits or colimits of all finite diagrams. See also Remark 3.4.

REMARK 3.3. One could take Parts (i) and (ii) as the definition of limit (respectively, colimit) computation. However, in order that our notion of categorical complexity is closer to the classical notions-such as circuit 
complexity in certain relevant categories (see Section 4), we also consider the constructivity condition. This roughly means that the limit (or colimit) computation does not forget how an object was constructed. It also prevents objects of exponential rank/size from being constructed; cf. Example 3.16.

REMARK 3.4. Of course, one may not be able to obtain every object, morphism, or diagram from a given set of basic morphisms $\mathcal{A}$ in a category $\mathcal{C}$. We will think of such objects/morphisms/diagrams as noncomputable in $\mathcal{C}$ with respect to $\mathcal{A}$.

We now describe a basic syntax for writing down the limit or colimit computations. The computation is described by a set expressions, each expression in a line. The first kind of expression is of the form

$$
\text { i. source, } f \text {, target }
$$

and describes objects and/or morphisms that are added to $D_{0}$. Here, $i$ is an identifier that can be any string. In subsequent lines, the identifier ' $i$ ' is used to refer to the source, and ' $i$ ', is used to refer to the target of the basic morphism $f \in \mathcal{A}$ that is added to $D_{0}$ by this expression. source and target are the identifiers of the vertices that are the intended source and target of the new morphism being attached to $D_{0}$.

If the source is a new vertex that did not exist in the diagram before, then we write $i . i, f$, target, or $i ., f$, target for it. If only the target is new, we write $i$. source, $f, i^{\prime}$ or $i$. source, $f, \ldots$; we write i. i, $f, i^{\prime}$ or $i ._{-}, f,_{-}$if both are new, distinct vertices, and $i ._{-}, f, i$ if both are new and are the same vertex.

There is no need to list all the morphisms in $D_{0}$ at the beginning, so we will have these steps as intermediate steps as well; as long as the morphisms attach only to other vertices in $D_{0}$, they be can be considered as part of $D_{0}$.

The second kind of expression is that of the form

$$
\text { i. } \lim (a, b, \ldots)
$$

which describes steps where a limit is added to the subdiagram. The identifiers $a, b, \ldots$ describe the vertices in the subdiagram whose limit is being taken. In subsequent steps, $i$ is used to refer to the limit added. Similarly, we write i. $\operatorname{colim}(a, b, \ldots)$ for describing colimit computations. We may use the notation $i->a$ to refer to morphisms created during the computation.

We start with two basic examples about constructions in the category of sets.

EXAmple 3.5. Let $\mathcal{C}$ be the category of sets and let $\mathcal{A}$ consist of a single morphism id $:\{1\} \rightarrow\{1\}$. Consider the colimit computation described by 


$$
\begin{array}{ll}
1 . & -,\{1\} \stackrel{\text { id }}{\rightarrow}\{1\}, 1 \\
2 . & -,\{1\} \stackrel{\text { id }}{\rightarrow}\{1\}, 2 \\
\ldots & \\
\text { n. } & \quad,\{1\} \stackrel{\text { id }}{\rightarrow}\{1\}, n \\
n+1 . & \operatorname{colim}(1,2, \ldots, n)
\end{array}
$$

For each $k \leqslant n$, the step $\mathrm{k} . \ldots,\{1\} \stackrel{\text { id }}{\rightarrow}\{1\}, \mathrm{k}$ is adding a new copy of $\{1\}$ to the diagram (that is, the vertex labeled $\mathrm{k}$ with a self-loop corresponding to id). In the end, $\mathrm{n}+1$ is the set with $n$ elements.

EXAMPLE 3.6. Continuing with the previous example, we now make a colimit computation that produces the morphism $\{0,1,2\} \stackrel{f}{\rightarrow}\{0,1,2\}$ in the category of sets, where $f(0)=0, f(1)=0, f(2)=1$.

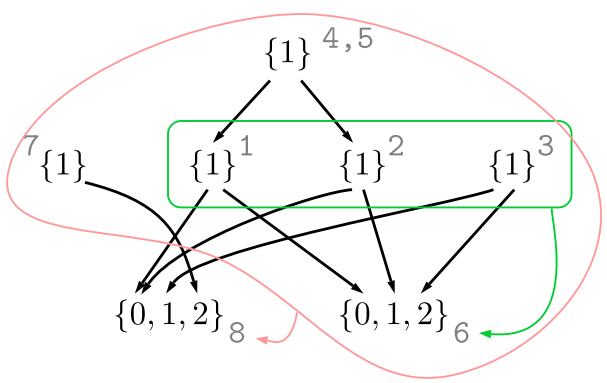
1. $\quad,\{1\} \stackrel{\text { id }}{\rightarrow}\{1\}, 1$
2. $\quad,\{1\} \stackrel{\text { id }}{\rightarrow}\{1\}, 2$
3. $\quad,\{1\} \stackrel{\text { id }}{\rightarrow}\{1\}, 3$
4. $\quad .,\{1\} \stackrel{\text { id }}{\rightarrow}\{1\}, 1$
5. $4,\{1\} \stackrel{\text { id }}{\rightarrow}\{1\}, 2$
6. $\operatorname{colim}(1,2,3)$
7. $\quad,\{1\} \stackrel{\text { id }}{\rightarrow}\{1\}, 7$
8. $\operatorname{colim}(1,2,3,4,6,7)$

The morphism $6->8$ is $f$, in the sense that the full subdiagram containing 6 and 8 is isomorphic to $\{0,1,2\} \stackrel{f}{\rightarrow}\{0,1,2\}$.

We will come back to sets later. We now discuss a more detailed example where we annotate each step in the computation.

EXAMPLE 3.7. Consider the category Vect Ve $_{k}$ vector spaces over a field $k$. Let $\mathcal{A}$ consist of the scalar multiplication morphisms $k \stackrel{c}{\rightarrow} k$ for each $c \in k$, the addition morphism $k^{2} \stackrel{+}{\rightarrow} k$, the two projections $\pi_{1}, \pi_{2}: k^{2} \rightarrow k$, and morphisms $0 \rightarrow k$, $k \rightarrow 0$. Say, the characteristic of $k$ is 0 and we wish to compute the morphism $f: k^{3} \rightarrow k^{2}, f(x, y, z)=(2 x+2 y+3 z, y+z)$. We describe the computation as follows. 


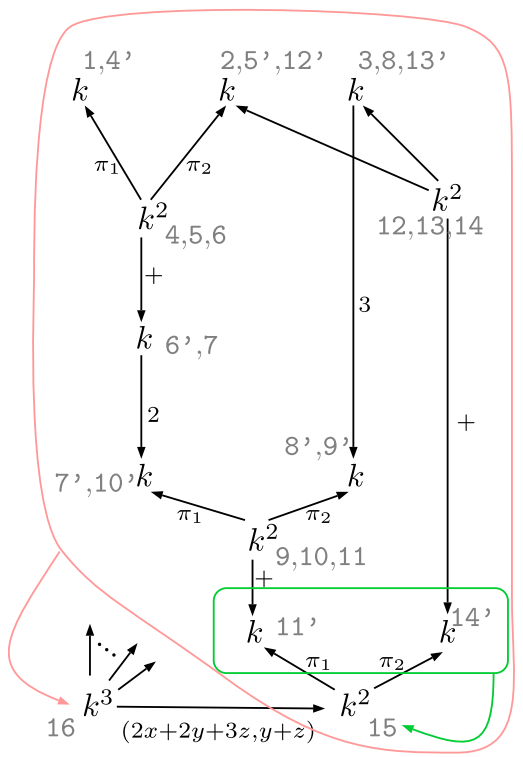
1. $\ldots, k \stackrel{1}{\rightarrow} k, 1$
2. $\ldots, k \stackrel{1}{\rightarrow} k, 2$
3. $-, k \stackrel{1}{\rightarrow} k, 3$
4. $-, k \times k \stackrel{\pi_{1}}{\longrightarrow} k, 1$
5. $4, k \times k \stackrel{\pi_{2}}{\longrightarrow} k, 2$
6. $4, k \times k \stackrel{+}{\rightarrow} k, 6$,
7. 6', $k \stackrel{2}{\rightarrow} k, 7$ '
8. $3, k \stackrel{3}{\rightarrow} k, 8$ '
9. $\ldots, k \times k \stackrel{\pi_{2}}{\longrightarrow} k, 8$,
10. $9, k \times k \stackrel{\pi_{1}}{\longrightarrow} k, 7$,
11. $9, k \times k \stackrel{+}{\rightarrow} k, 11$,
12. $-, k \times k \stackrel{\pi_{1}}{\longrightarrow} k, 2$
13. $12, k \times k \stackrel{\pi_{2}}{\longrightarrow} k, 3$
14. $12, k \times k \stackrel{+}{\rightarrow} k, 14$ '
15. $\lim \left(11^{\prime}, 14^{\prime}\right)$
16. $\lim (1,1,, 2,2, \ldots$
$\ldots, 14,14,, 15)$

REMARK 3.8. Two facts about limits and colimits are useful in thinking about the above example and other computations.

The first (as already noted in Example 2.13) is that if $f: X \rightarrow Y$ and $g: Y \rightarrow$ $Z$ are morphisms, then the limit of the diagram $X \stackrel{f}{\rightarrow} Y \stackrel{g}{\rightarrow} Z$ is (isomorphic to) $X$, and the induced morphism $X \rightarrow Z$ is equal to the composition $g \circ f$. So, compositions are obtained using limits. The second fact is the following. Let $D: I=(V, E, s, t) \rightarrow \mathcal{C}$ be a diagram, and $L=\lim D$, and let $\varphi: C \rightarrow D$ be a cone over $D$, where $C$ is a constant diagram of shape $I$ and value $X$. Let $I^{\prime}=\left(V^{\prime}, E, s^{\prime}, t^{\prime}\right)$ be the graph, with $V^{\prime}=V \cup\left\{v_{0}, v_{1}\right\}, E^{\prime}=E \cup \bigcup_{v \in V}\left\{e_{v}, e_{v}^{\prime}\right\}$,

$$
\left.\begin{array}{l}
s^{\prime}(e)=s(e), \\
t^{\prime}(e)=t(e),
\end{array}\right\} \quad \text { for } e \in E,
$$

$$
\left.\left.\left.\begin{array}{l}
s^{\prime}(e)=v_{0}, \\
t^{\prime}(e)=v,
\end{array}\right\} \quad \text { for } e=e_{v}, \begin{array}{l}
s^{\prime}(e)=v_{1}, \\
t^{\prime}(e)=v,
\end{array}\right\} \quad \text { for } e=e_{v}^{\prime},\right\} \quad \text { for } v \in V
$$

and $D^{\prime}: I^{\prime} \rightarrow \mathcal{C}$ be the diagram defined by,

$$
\begin{aligned}
& D^{\prime}(v)=D(v), \quad \text { for } v \in V, \\
& D^{\prime}\left(v_{0}\right)=L, \\
& D^{\prime}\left(v_{1}\right)=X,
\end{aligned}
$$




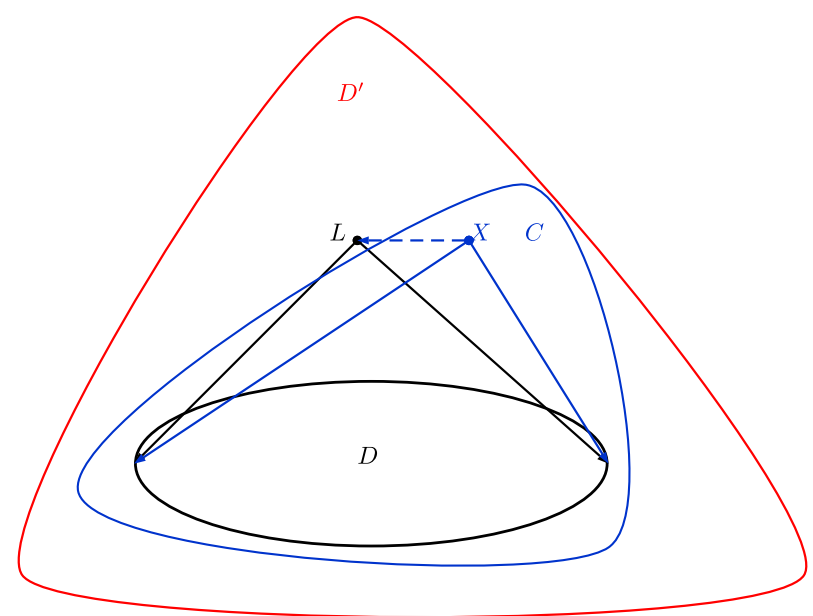

Figure 2. One extra limit.

$$
\begin{aligned}
& D^{\prime}(e)=D(e), \quad \text { for } e \in E, \\
& D^{\prime}(e)=(\lim D)_{v}, \quad \text { for } e=e_{v}, v \in V, \\
& D^{\prime}(e)=\varphi_{v}, \quad \text { for } e=e_{v}^{\prime}, v \in V .
\end{aligned}
$$

Then, $\lim D^{\prime}$ is isomorphic to $X$, and morphism $X \rightarrow L$ in this corresponding limit cone is the unique morphism coming from the universal property of the limit $L$. Thus, in a limit computation, once the cones corresponding to $L$ and $X$ are computed, in order to obtain the morphism $X \rightarrow L$, which is implied by the universal property of limits, one needs to take just one additional limit.

A similar fact holds for colimits (with the arrows reversed). In other words, let $D: I=(V, E, s, t) \rightarrow \mathcal{C}$ be a diagram, and $M=\operatorname{colim} D$, and let $\varphi: D \rightarrow C$ be a cocone over $D$, where $C$ is a constant diagram of shape $I$ and value $Y$. Then, in a colimit computation, once the cocones corresponding to $M$ and $Y$ are computed, in order to obtain the morphism $M \rightarrow Y$, which is implied by the universal property of colimits, one needs to take just one additional colimit.

3.2. Mixed limit-colimit computations. We now discuss computations where we can use limits and colimits together. We call these mixed computations.

Definition 3.9. A mixed computation is a finite sequence $\left(D_{0}, \ldots, D_{s}\right)$ of diagrams with $D_{i}: I_{i} \rightarrow U(\mathcal{C})$, where $D_{0}$ consists only of morphisms in $\mathcal{A}$, and for each $i=1, \ldots, s, D_{i}$ is obtained from $D_{i-1}$ by adding either the limit of a subdiagram with the corresponding cone morphisms or colimit of a subdiagram with the corresponding cocone morphisms. 
Note that there is no constructivity assumption for mixed computations. To include it would have been too restrictive and would have prevented natural applications like gluing geometric objects already constructed.

EXAMPLE 3.10 (Monotone Boolean circuits). Let $\mathcal{B}_{n}$ be the lattice of subsets of $\{0,1\}^{n}$, which is a category whose objects are the subsets of $\{0,1\}^{n}$, and with $\operatorname{Hom}_{\mathcal{B}_{n}}(A, B)=\{\iota\}$ if $A \subset B$, where $\iota: A \rightarrow B$ is the inclusion, and $\operatorname{Hom}_{\mathcal{B}_{n}}(A$, $B)=\emptyset$ otherwise. Let $Z_{i}=\left\{\left(x_{1}, \ldots, x_{n}\right) \in\{0,1\}^{n} \mid x_{i}=1\right\}$. Let $\mathcal{A}_{n}$ be the set of basic morphisms $\left\{\operatorname{id}_{Z_{i}} \mid i=1, \ldots, n\right\}$. Let $\mathcal{B}=\bigsqcup_{n=1}^{\infty} \mathcal{B}_{n}$ be the disjoint union of these categories and $\mathcal{A}=\coprod_{n=1}^{\infty} \mathcal{A}_{n}$.

We show that there is a correspondence between multi-output monotone Boolean circuits with $n$ inputs and mixed computations in $\mathcal{B}_{n}$. Given a monotone Boolean circuit, consider the corresponding straight-line program with Boolean operations. Start a mixed computation in $\mathcal{B}_{n}$ with a copy of each of the subsets $Z_{i}$. These correspond to the input variables $z_{1}, \ldots, z_{n}$ of the straight-line program. Subsequent entries $z_{n+1}, z_{n+2} \ldots$ will correspond to newly constructed objects in the mixed computation. For each operation in the straight-line program of the form $z_{i}=z_{j} \wedge z_{k}$, take the limit of the objects corresponding to $z_{j}$ and $z_{k}$; similarly, take the colimit for $z_{i}=z_{j} \vee z_{k}$. To make a straight-line program from a mixed computation, start with the input variables $z_{1}, \ldots, z_{n}$ and add $k-1$ new $\wedge$ operations for each limit of $k$ objects and $k-1$ new $\vee$ operations for each colimit of $k$ objects (ignoring the arrows does not change the limit/colimit).

Thus, mixed computations in $\mathcal{B}_{n}$ are in direct correspondence with monotone straight-line programs or, equivalently, monotone Boolean circuits.

EXAmPLE 3.11. Consider the category Top of topological spaces. Let $I=[0$, 1] be the unit interval, and let the basic morphisms consist of $I \stackrel{\text { id }}{\rightarrow} I, I \rightarrow p t$, $p t \stackrel{0}{\rightarrow} I$, and $p t \stackrel{1}{\rightarrow} I$. We can build all cubes using limits and then can glue these using colimits to construct many topological spaces.

We will reconsider mixed computations when we look at the complexity of projective schemes.

3.3. Cost and complexity. Let $c_{0}: \mathcal{A} \rightarrow \mathbb{Z}_{\geqslant 0}$ be any function, considered as the cost of the basic morphisms.

Definition 3.12. The cost of the computation $\left(D_{0}, \ldots, D_{s}\right)$ is the number of steps plus the cost of the initial diagram $D_{0}$ consisting of basic morphisms, that is,

$$
c\left(D_{0}, \ldots, D_{s}\right)=s+\sum_{f \in \operatorname{edges}\left(I_{0}\right)} c_{0}\left(D_{0}(f)\right) .
$$


If $c_{0}$ is not specified, then we consider it to be the constant function 1 , so every basic morphism will have unit cost. This will be the case in almost every example we consider.

DEFINITION 3.13. The limit (respectively, colimit; respectively, mixed) complexity, $c_{\mathcal{C}, \mathcal{A}, c_{0}}^{\lim }(D)$ (respectively, $c_{\mathcal{C}, \mathcal{A}, c_{0}}^{\text {colim }}(D)$; respectively, $c_{\mathcal{C}, \mathcal{A}, c_{0}}^{\text {mixed }}(D)$ ), of a diagram $D$ in a category $\mathcal{C}$ is the cost of the limit (respectively, colimit; respectively, mixed) computation using basic morphisms $\mathcal{A}$, which has the smallest cost among all such computations that compute $D$. When $c_{0}$ is the constant function 1 , we will omit it from the subscript and just write $c_{\mathcal{C}, \mathcal{A}}^{\lim }(D)$, $c_{\mathcal{C}, \mathcal{A}}^{\text {colim }}(D)$, or $c_{\mathcal{C}, \mathcal{A}}^{\text {mixed }}(D)$.

For a morphism $f: X \rightarrow Y$ in $\mathcal{C}$, the complexity $c(f)$ of $f$ is the complexity of the corresponding diagram mapping two objects and a single morphism $X \stackrel{f}{\rightarrow} Y$. For an object $X$ in $\mathcal{C}$, the complexity $c(X)$ of $X$ is the complexity of the diagram with one object, $X$.

EXAMPLE 3.14 (Gluing simplices). Let $\mathcal{C}=$ Top be the category of topological spaces and let $\mathcal{A}$ be the set of all face embeddings $\Delta_{n} \hookrightarrow \Delta_{m}$ corresponding to each strictly increasing map $[n] \rightarrow[m]$, where $\Delta_{n}$ is the standard $n$-simplex. Colimit computations correspond to gluing operations between simplices. The colimit complexity then measures how many simplices are needed to construct a given topological space by gluing.

We now go back to considering $\mathcal{C}=$ Set with the basic morphisms $\mathcal{A}$ consisting of a single morphism id $:\{1\} \rightarrow\{1\}$.

Proposition 3.15 (Colimit complexity of sets). In the category Set, let

$$
\mathcal{A}=\{\mathrm{id}:\{1\} \rightarrow\{1\}\}, c_{0}(\mathrm{id})=1 .
$$

Then, for any set finite set $S$,

$$
c_{\text {Set }, \mathcal{A}}^{\text {colim }}(S)=\operatorname{card}(S)+1 .
$$

Proof. Since finite sets of equal size are isomorphic, a computation will compute $S$ if and only if it computes any set of cardinality equal to card $(S)$. As in Example 3.5, starting with $\operatorname{card}(S)$ copies of $\{1\}$ and taking their colimit, we get a set of cardinality $\operatorname{card}(S)$. So, the complexity is bounded from above by $\operatorname{card}(S)+1$. To see that this is the most efficient way of producing a set with $\operatorname{card}(S)$ elements, we use Lemma 3.19, which states that if we only care about building a single object, then a colimit computation can be replaced by a single 
colimit on $D_{0}$ consisting of basic morphisms. Since the identity on $\{1\}$ is the only basic morphism in this case, taking the colimit of $\operatorname{card}(S)$ copies of $\{1\}$ is the most efficient way to obtain an object isomorphic to $S$.

EXAMPLE 3.16 (Nonconstructive colimit complexity). The following example shows the difference between colimit computations and nonconstructive computations. Consider the computation

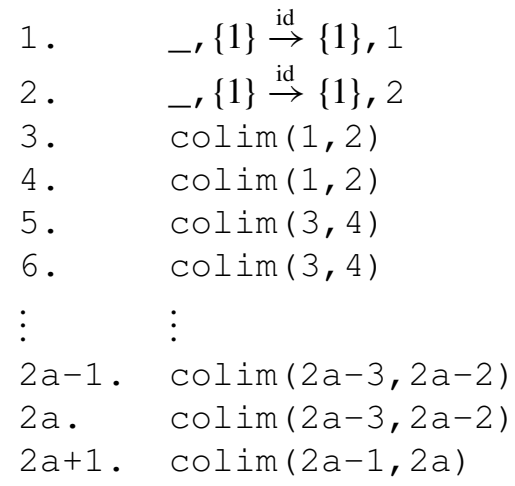

This computation produces a set of size $2^{a}$. Observe that the steps 5 to $2 \mathrm{a}+1$ are not constructive. To make them constructive, one would need to include 1 , $\ldots 2 i-2$ in the colimit in step $2 i-1,3 \leqslant i \leqslant a+1$, and include $1, \ldots 2 i-2$ in the colimit in step $2 i, 3 \leqslant i \leqslant a$. This would produce in step $2 a+1$ a set with just two elements.

EXAMPLE 3.17 (Nonconstructive mixed complexity). Using mixed computations, one can construct a set of doubly exponential complexity. Consider for example the following mixed computation.

$\begin{array}{ll}\text { 1. } & \quad,\{1\} \stackrel{\text { id }}{\rightarrow}\{1\}, 1 \\ 2 . & \quad,\{1\} \stackrel{\text { id }}{\rightarrow}\{1\}, 2 \\ 3 . & \operatorname{colim}(1,2) \\ 4 . & \operatorname{colim}(1,2) \\ 5 . & \lim (3,4) \\ 6 . & \lim (3,4) \\ \vdots & \vdots \\ 2 a-1 . & \lim (2 a-3,2 a-2) \\ 2 a . & \lim (2 a-3,2 a-2) \\ 2 a+1 . & \lim (2 a-1,2 a)\end{array}$


It is easy to check that it produces a set of cardinality $2^{2^{a}}$. So the mixed complexity of a finite set $\operatorname{card}(S)$ is $\mathrm{O}(\log \log \operatorname{card}(S))$.

One important feature of categorical complexity is that it allows one to define complexity of not just single objects or even morphisms of a given category (equipped with a set of basic morphisms), but one has a notion of complexity of arbitrary (finite) diagrams of the category as well. This last notion has no analog in classical theory of computational complexity.

We illustrate this feature in the following simple example.

EXAMPLE 3.18. We will consider the colimit complexity (in the category Vect $_{k}$, where $k$ is a field) of two diagrams consisting of the inclusion morphisms of $n^{2}$ different subspaces of dimension two in a $2 n$-dimensional $k$-vector space.

In the first diagram, the subspaces are assumed to be generic (Case (a) below), and we prove that the colimit complexity of the diagram is in $O\left(n^{3}\right)$ (which agrees with the intuition that to specify $n^{2}$ inclusions of two-dimensional subspaces in a $2 n$-dimensional vector space, we need to specify a matrix in $\left.k^{2 n \times 2 n^{2}}\right)$.

In the second diagram, the subspaces are in a special position (Case (b) below), and we prove that the colimit complexity of the diagram is in $O\left(n^{2}\right)$ (intuitively, only $2 n$ distinct columns appear in the $2 n \times 2 n^{2}$ corresponding to the inclusion if the basis vectors are chosen properly). Categorical complexity helps in quantifying the distinction in the complexity of the two diagrams having the same shape. There is no analog in classical computational complexity, which deals mainly with membership questions (and thus from the point of view of category theory complexities of objects rather than general diagrams) of this kind of distinction.

We consider the category Vect $_{k}$ of vector spaces over a field $k$ and let $\mathcal{A}$ consist of the scalar multiplication morphisms $k \stackrel{c}{\rightarrow} k$ for each $c \in k$, the addition morphism $k^{2} \stackrel{+}{\rightarrow} k$, the two projections $\pi_{1}, \pi_{2}: k^{2} \rightarrow k$, and morphisms $0 \rightarrow k$, $k \rightarrow 0$ (as in Example 3.7).

(a) Let $n \geqslant 2, S_{1}, \ldots, S_{n^{2}} \subset V$ be subspaces of a finite-dimensional $k$-vector space $V$, and suppose that $\operatorname{dim} V=2 n, \operatorname{dim} S_{i}=2,1 \leqslant i \leqslant n^{2}$, and $\operatorname{dim}\left(S_{i} \cap S_{j}\right)=0,1 \leqslant i<j \leqslant n^{2}$.

Consider the diagram shown in Figure 3 , where the $\phi_{i}, 1 \leqslant i \leqslant n^{2}$, are the inclusion homomorphisms.

We now show how to produce the above diagram in Figure 3 using a colimit computation. 


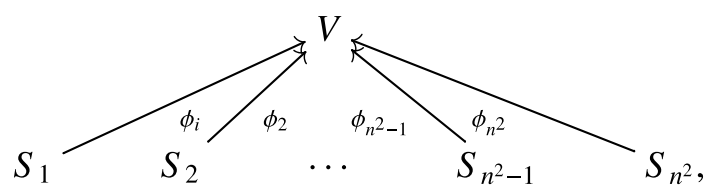

Figure 3. Subspace diagram.

Let for each $i, 1 \leqslant i \leqslant n^{2}, S_{i}^{\prime}$, $S_{i}^{\prime \prime}$ be one-dimensional subspaces of $S_{i}$ such that $S_{i}=S_{i}^{\prime} \oplus S_{i}^{\prime \prime}$, and let $\psi_{i}^{\prime}, \psi_{i}^{\prime \prime}$ denote the inclusions of $S_{i}^{\prime}, S_{i}^{\prime \prime}$ into $V$, respectively.

Similarly, let $E_{1}, \ldots, E_{2 n} \subset V$ be one-dimensional subspaces of $V$ such that $V=E_{1} \oplus \cdots \oplus E_{2 n}$. Then, $V$ is canonically isomorphic to the direct product $E_{1} \times \cdots \times E_{2 n}$ and let $\pi_{i}: V \rightarrow E_{i}$ denote the canonical projections.

For each $1 \leqslant i \leqslant 2 n, 1 \leqslant j \leqslant n^{2}$, we first use colimit computation to obtain a diagram computing the $2 n^{3}$ linear morphisms, $\phi_{i, j}=\pi_{i} \circ\left(\psi_{j}^{\prime} \oplus \psi_{j}^{\prime \prime}\right)$ : $S_{j}^{\prime} \oplus S_{j}^{\prime \prime} \rightarrow E_{i}$, to obtain the diagram shown in Figure 4.

The colimit computation to obtain the diagram shown in Figure 4 is as follows. For each $j, 1 \leqslant j \leqslant n$, first take the colimit of $S_{j}^{\prime}$ and $S_{j}^{\prime \prime}$ to obtain the diagram

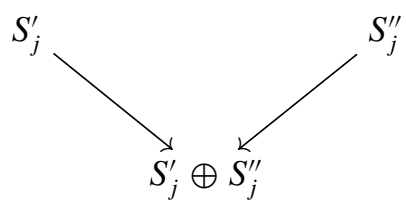

and then successively for each $i, 1 \leqslant i \leqslant n$, take colimits of the diagrams

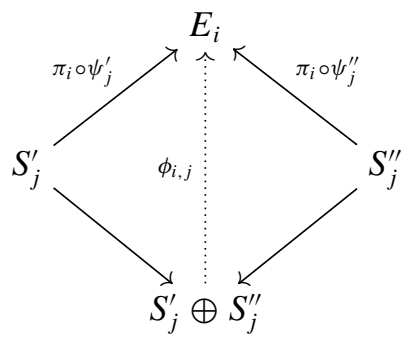

and observe (cf. Remark 3.8) that we obtain the morphisms $\phi_{i, j}$. Note that the morphisms $\pi_{i} \circ \psi_{j}^{\prime}, \pi_{i} \circ \psi_{j}^{\prime \prime}$ being morphisms between one-dimensional $k$-vector spaces are basic. It is easy to check that the total cost of the colimit computation described above is

$$
2 n^{3}+n^{2}+2 n^{3}=4 n^{3}+n^{2} .
$$




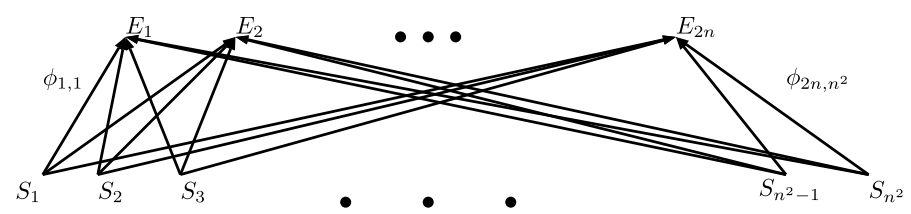

Figure 4. Subspace diagram before taking the last colimit.

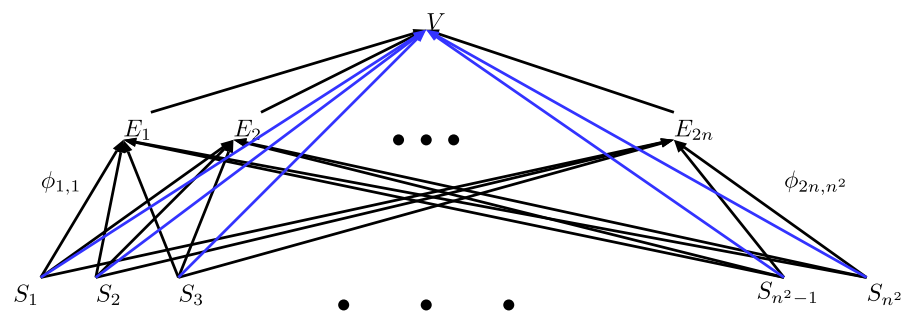

Figure 5. Diagram after taking the last colimit.

We then take the colimit of the whole diagram computed till now (as shown in Figure 5), and obtain the desired diagram as a subdiagram (whose morphisms are shown in blue in Figure 5).

The total cost is $4 n^{3}+n^{2}+1=O\left(n^{3}\right)$.

(b) We now consider subspaces $S_{1}, \ldots, S_{n^{2}}$ in a special position, and prove that colimit complexity of the corresponding diagram can be much smaller.

Let $V=V^{\prime} \oplus V^{\prime \prime}$, where $\operatorname{dim} V^{\prime}=\operatorname{dim} V^{\prime \prime}=n$. Let $L_{i}^{\prime} \subset V^{\prime}, L_{i}^{\prime \prime} \subset V^{\prime \prime}$, $1 \leqslant i \leqslant n$ be subspaces with $\operatorname{dim} L_{i}^{\prime}=\operatorname{dim} L_{i}^{\prime \prime}=1,1 \leqslant i \leqslant n$, and suppose that $S_{1}, \ldots, S_{n^{2}}$ are the subspaces $L_{i}^{\prime} \oplus L_{j}^{\prime \prime} \subset V=V^{\prime} \oplus V^{\prime \prime}, 1 \leqslant i, j \leqslant n$.

Let $V^{\prime}$ (respectively, $V^{\prime \prime}$ ) be the direct sum of one-dimensional subspaces $E_{1}^{\prime}, \ldots, E_{n}^{\prime}$ (respectively, $\left.E_{1}^{\prime \prime}, \ldots, E_{n}^{\prime \prime}\right)$. Then $V^{\prime}=E_{1}^{\prime} \oplus \cdots \oplus E_{n}^{\prime}, V^{\prime \prime}=E_{1}^{\prime \prime} \oplus$ $\cdots \oplus E_{n}^{\prime \prime}$, and moreover, $V^{\prime}$ (respectively, $V^{\prime \prime}$ ) is also canonically isomorphic to the direct product $E_{1}^{\prime} \times \cdots \times E_{n}^{\prime}$ (respectively, $E_{1}^{\prime \prime} \times \cdots \times E_{n}^{\prime \prime}$ ). Let for $1 \leqslant i \leqslant n, \pi_{i}^{\prime}: V^{\prime} \rightarrow E_{i}^{\prime}$ (respectively, $\pi_{i}^{\prime \prime}: V^{\prime \prime} \rightarrow E_{i}^{\prime \prime}$ ) denote the canonical projections. Also, for $1 \leqslant j \leqslant n$, let $\psi_{j}^{\prime}: L_{j}^{\prime} \rightarrow V^{\prime}$ (respectively, $\psi_{j}^{\prime \prime}: L_{j}^{\prime \prime} \rightarrow$ $V^{\prime \prime}$ ) denote the inclusion morphisms, and denote $\phi_{i, j}^{\prime}=\pi_{i}^{\prime} \circ \psi_{j}^{\prime}$ (respectively, $\left.\phi_{i, j}^{\prime \prime}=\pi_{i}^{\prime \prime} \circ \psi_{j}^{\prime \prime}\right)$.

In this case, we can construct the diagram in Figure 3 in the following way.

First construct the diagram containing the morphisms $\phi_{i, j}^{\prime}$ and $\phi_{i, j}^{\prime \prime}$ as shown in Figure 6. This costs $2 n^{2}$. 


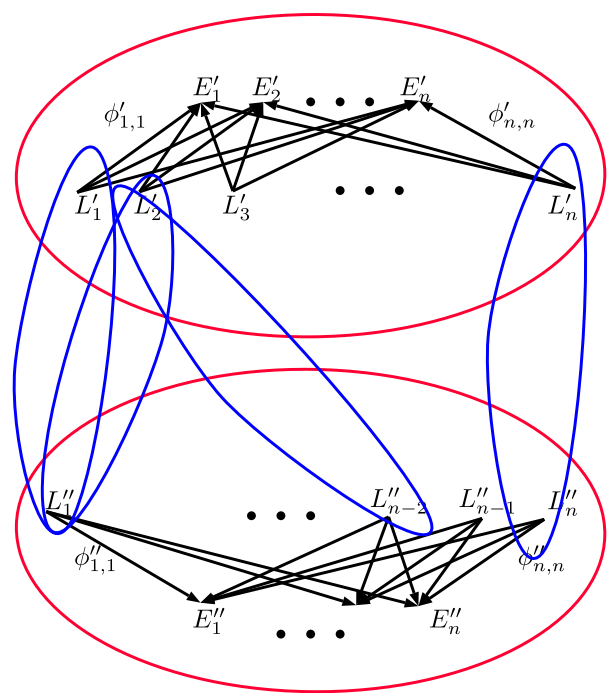

Figure 6. Colimit computation of arrangement of subspaces in a special position before taking any colimits.

Next take colimits of the $n^{2}$ subdiagrams circled in blue (corresponding to pairs of vertices $L_{i}, L_{j}^{\prime}, 1 \leqslant i, j \leqslant n$ ) to obtain the diagram shown in Figure 7 . Finally, take a colimit of the entire diagram constructed so far to obtain the diagram shown in Figure 8. It is an easy exercise to check that the required diagram occurs as a subdiagram (whose morphisms are shown using blue broken arrows in Figure 8) of the diagram so obtained. The total cost is $3 n^{2}+1=O\left(n^{2}\right)$.

Thus, while both arrangements of linear subspaces $S_{1}, \ldots, S_{n^{2}} \subset V$ in Parts (a) and (b) contain the same number of subspaces, and would require the same number of polynomials to define (say, using a first-order formula in the language of reals), the second one is 'simpler' than the first in terms of its categorical colimit complexity.

3.4. Useful facts about limit and colimit computations. We now collect a few facts that will be useful for proving statements about objects and morphisms computed by limit and colimit computations.

The following lemma, which was already used in the proof of Proposition 3.15, shows that if the aim is to produce a specific object, intermediate steps in a 


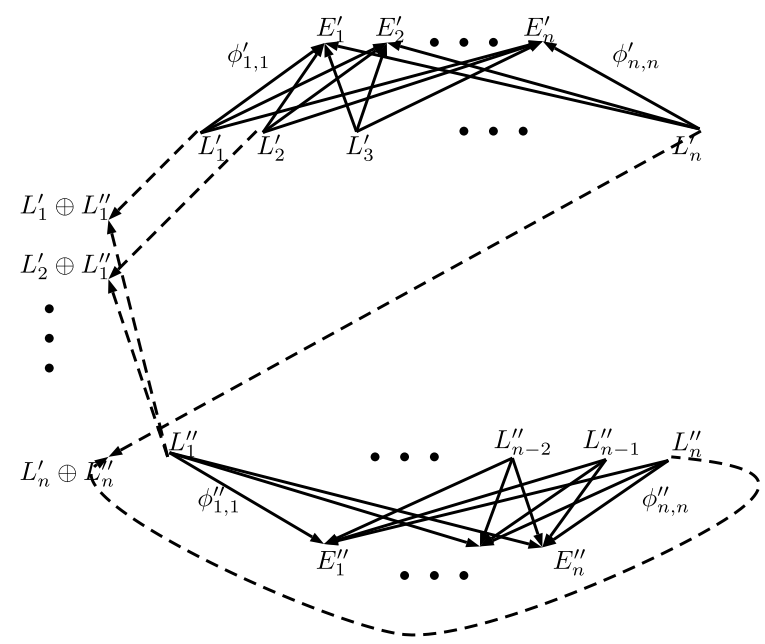

Figure 7. Colimit computation of arrangement of subspaces in a special position after taking $n^{2}$ colimits.

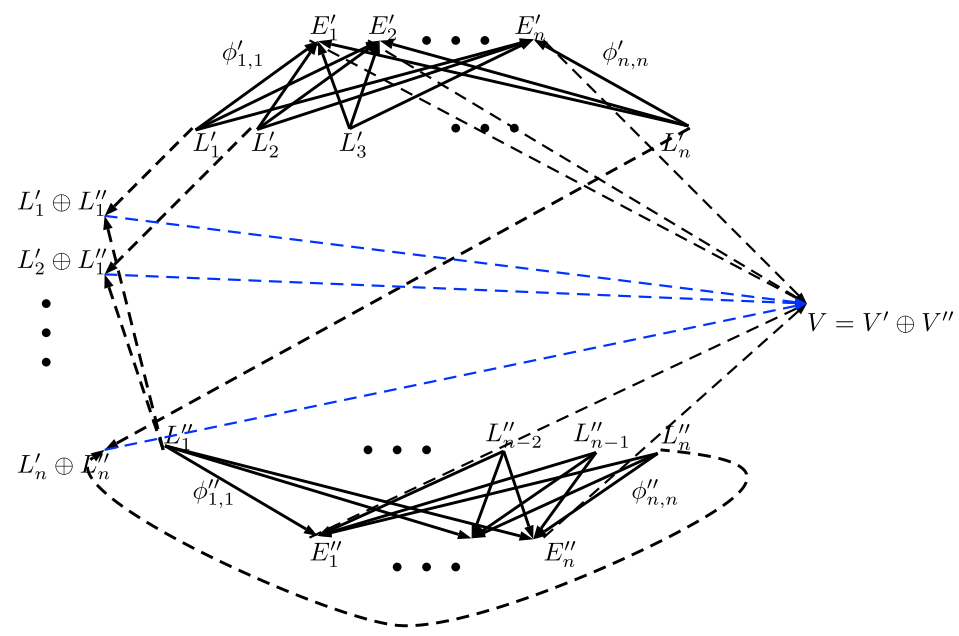

Figure 8. Colimit computation of arrangement of subspaces in a special position after taking the final colimit.

limit or colimit computation are unnecessary. The key here is the constructivity assumption. 
LemMa 3.19. Assume $\mathcal{C}$ has finite limits (respectively, colimits). An object produced in a limit computation (respectively, colimit computation) is a limit (respectively, colimit) of a diagram consisting only of basic morphisms.

Proof. Let $\left(D_{0}, \ldots, D_{s}\right)$ be a limit computation and let $X$ be an object appearing in $D_{s}$. The point of the statement is that constructivity ensures that the information that would be added in intermediate limits is also included in the final limit that would produce $X$.

More precisely, let $L_{i}=\left.\lim D_{i-1}\right|_{J_{i}}$ be the limit added to the diagram at the $i$ th step. Let $J_{i}^{\prime}=I_{0} \cap J_{i}$. So we have that $\left.D_{i-1}\right|_{J_{i}^{\prime}}$ is the portion of the subdiagram of $\left.D_{i-1}\right|_{J_{i}}$, which is also in $D_{0}$. We claim that $\left.L_{i} \cong \lim D_{i-1}\right|_{J_{i}^{\prime}}$. Indeed, the universal property of limits and constructivity imply that cones from any object $Z$ to $\left.D_{i-1}\right|_{J_{i}^{\prime}}$ can be uniquely extended to cones from $Z$ to $\left.D_{i-1}\right|_{J_{i}}$, and therefore $\left.\lim D_{i-1}\right|_{J_{i}^{\prime}}$ satisfies the same universal property as $L_{i}$.

The analogous proof holds for colimits.

The following remarks are very useful for working with objects and morphisms produced in limit computations.

REMARK 3.20. If the category $\mathcal{C}$ has finite products and equalizers, then we can write any limit $L$ as an equalizer; see for example, [Awo10, 5.4]. More precisely, suppose that $J=(V, E, s, t)$ is a finite graph and $D: J \rightarrow U(\mathcal{C})$ a diagram. Consider the following diagram:

$$
\prod_{v \in V} D(v) \underset{\phi}{\stackrel{\psi}{\longrightarrow}} \prod_{e \in E} D(t(e)),
$$

where $\phi$ is the canonically defined morphism from $\prod_{v \in V} D(v)$ to the product $\prod_{e \in E} D(t(e))$ induced from the morphisms

$$
\left(\operatorname{pr}_{t(e)}: \prod_{v \in V} D(v) \rightarrow t(e)\right)_{e \in E},
$$

and $\psi$ is the canonically defined morphism from $\prod_{v \in V} D(v)$ to the product $\prod_{e \in E} D(t(e))$ induced from the morphisms

$$
\left(D(e) \circ \operatorname{pr}_{s(e)}: \prod_{v \in V} D(v) \rightarrow t(e)\right)_{e \in E},
$$

with both maps being defined by the universal property of products. 
Let $L \stackrel{f}{\rightarrow} \prod_{v \in V} D(v)$ in the following diagram be the equalizer of diagram (2):

$$
L \stackrel{f}{\longrightarrow} \prod_{v \in V} D(v) \underset{\phi}{\longrightarrow} \prod_{e \in E} D(t(e)) .
$$

Then the object $L$ along with the morphisms

$$
\left(\operatorname{pr}_{D(v)} \circ f: L \rightarrow D(v)\right)_{v \in V}
$$

is isomorphic to the $\operatorname{limit}, \lim D$, of $D$.

REMARK 3.21. If $X \stackrel{f}{\rightarrow} Y$ is a morphism computed by a limit computation, and neither of $X$ and $Y$ is in $D_{0}$, then $X$ must have been computed as a limit of a diagram that contains $Y$. By constructivity, the diagram whose limit is $X$ must contain the diagram that produced $Y$ as a subdiagram. Therefore, if $X=\lim D$, where $D:(V, E) \rightarrow U(\mathcal{C})$ is a diagram of basic morphisms, then $Y=\lim D^{\prime}$, where $D^{\prime}$ is the subdiagram corresponding to a full subgraph $\left(V^{\prime}, E^{\prime}\right) \subset(V, E)$. We then have a commuting diagram

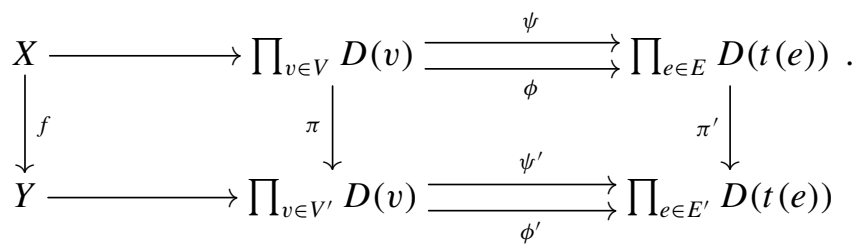

In particular, $f$ is induced by the projection $\pi$.

We have corresponding facts for colimits as well.

REMARK 3.22. If the category $\mathcal{C}$ has finite coproducts and coequalizers, then we can write any colimit $M$ as a coequalizer. More precisely, suppose that $J=(V$, $E, s, t)$ is a finite graph and $D: J \rightarrow U(\mathcal{C})$ is a diagram. Consider the following diagram:

$$
\bigsqcup_{e \in E} D(s(e)) \underset{\phi}{\longrightarrow} \bigsqcup_{v \in V} D(v),
$$

where $\phi$ is the canonically defined morphism from $\bigsqcup_{e \in E} D(s(e))$ to $\bigsqcup_{v \in V} D(v)$ induced from the morphisms

$$
\left(\mathrm{j}_{s(e)}: D(s(e)) \rightarrow \coprod_{v \in V} D(v)\right)_{e \in E},
$$


and $\psi$ is the canonically defined morphism from $\bigsqcup_{e \in E} D(s(e))$ to $\bigsqcup_{v \in V} D(v)$ induced from the morphisms

$$
\left(\mathrm{j}_{t(e)} \circ D(e): D(s(e)) \rightarrow \coprod_{v \in V} D(v)\right)_{e \in E},
$$

with both maps being defined by the universal property of coproducts.

Let $\bigsqcup_{v \in V} D(v) \stackrel{f}{\rightarrow} M$ in the following diagram be the coequalizer of diagram (3):

$$
\bigsqcup_{e \in E} D(s(e)) \underset{\phi}{\longrightarrow} \coprod_{v \in V} D(v) \stackrel{f}{\longrightarrow} M .
$$

Then the object $M$ with the morphisms

$$
\left(f \circ \mathrm{j}_{D(v)}: D(v) \rightarrow M\right)_{v \in V}
$$

is isomorphic to the to colimit, $\operatorname{colim} D$, of $D$.

REMARK 3.23. If $Y \stackrel{f}{\rightarrow} X$ is a morphism computed by a colimit computation, and neither of $X$ and $Y$ is in $D_{0}$, then $X$ must have been computed as a colimit of a diagram that contains $Y$. By constructivity, the diagram whose colimit is $X$ must contain the diagram that produced $Y$ as a subdiagram. Therefore, if $X=\operatorname{colim} D$, where $D:(V, E) \rightarrow U(\mathcal{C})$ is a diagram of basic morphisms, then $Y=\operatorname{colim} D^{\prime}$, where $D^{\prime}$ is the subdiagram corresponding to a full subgraph $\left(V^{\prime}, E^{\prime}\right) \subset(V, E)$. We then have a commuting diagram

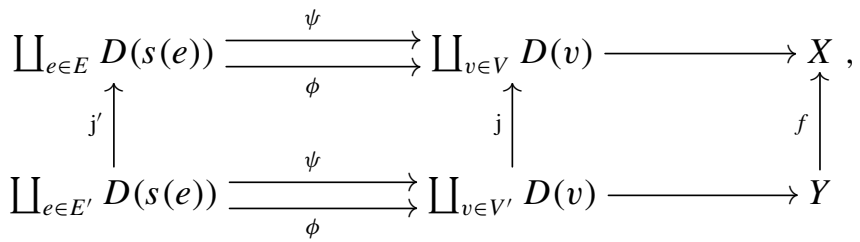

where $\mathrm{j}, \mathrm{j}^{\prime}$ are the morphisms induced by the universal property of coproducts. In particular, $f$ is induced by the morphism $\mathrm{j}$.

\section{Diagram computations, circuits, and algebraic varieties}

We now make a comparison between the arithmetic circuit complexity of polynomials and the limit complexity of the varieties, which are their zero-sets. For simplicity, we will start with the category of affine algebraic varieties, but what we describe will make sense in other settings like affine schemes and algebras. 
4.1. Limit computations in affine varieties, schemes, and $\boldsymbol{k}$-algebras. Let $\mathcal{C}$ be the category $\mathbf{A f f} \mathbf{V a r}_{k}$ of affine algebraic varieties over a field $k$. Let $\mathcal{A}$ consist of the following basic morphisms.

$$
\begin{aligned}
& \mathbb{A}^{1} \stackrel{c}{\rightarrow} \mathbb{A}^{1}, \quad \text { for each } c \in k, \\
& \mathbb{A}^{1} \times \mathbb{A}^{1} \stackrel{+}{\rightarrow} \mathbb{A}^{1}, \\
& \mathbb{A}^{1} \times \mathbb{A}^{1} \stackrel{\times}{\rightarrow} \mathbb{A}^{1}, \\
& \mathbb{A}^{1} \times \mathbb{A}^{1} \stackrel{\pi_{1}, \pi_{2}}{\rightarrow} \mathbb{A}^{1}, \\
& \mathbb{A}^{1} \rightarrow \mathbb{A}^{0}, \quad \text { and } \mathbb{A}^{0} \stackrel{c}{\rightarrow} \mathbb{A}^{1}, \quad \text { for each } c \in k .
\end{aligned}
$$

Each of these morphisms is considered to have unit cost.

EXAMPLE 4.1. As an example, let us make a limit computation of the morphism $\mathbb{A}^{3} \stackrel{x^{2}+y z}{\longrightarrow} \mathbb{A}^{1}$ using these basic morphisms.

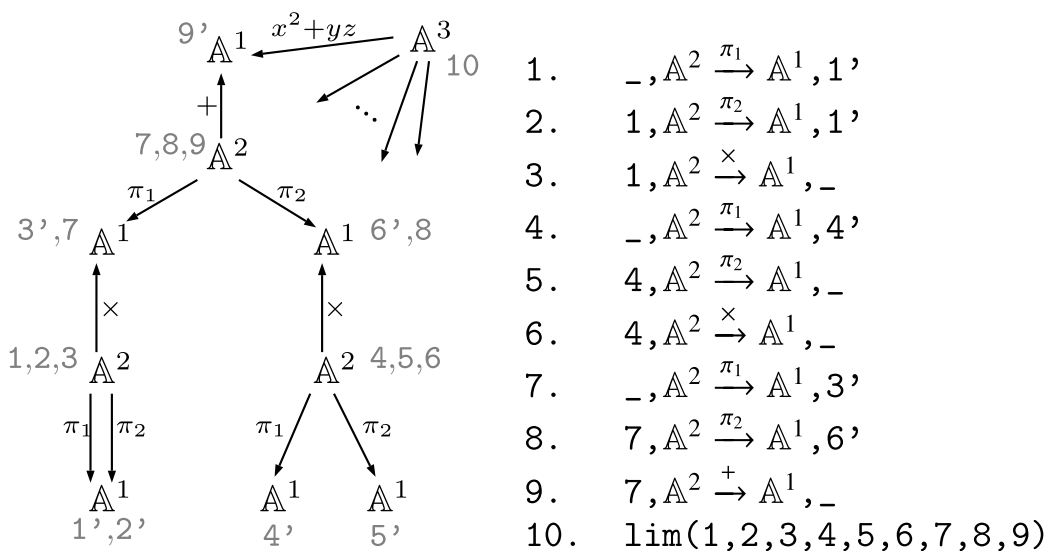

The following shows that a similar computation can be done to compute any polynomial map.

THEOREM 4.2. Let $f \in k\left[x_{1}, \ldots, x_{n}\right]$ be a polynomial of degree $d$. Assume that $f$ is computed by a straight-line program (cf. [Bür00, Definition 2.1]) $\Gamma$ of length $N$. Then, the limit complexity (and therefore the categorical complexity) of the zero-set $X \subset \mathbb{A}^{n}$ of $f$ is in $\mathrm{O}(N)$. 
Proof. Using the operations in the straight-line program $\Gamma$, we will construct a morphism $\mathbb{A}^{n} \stackrel{f}{\rightarrow} \mathbb{A}^{1} . X$ is then the limit $\mathbb{A}^{n} \stackrel{f}{\rightarrow} \mathbb{A}^{1} \stackrel{0}{\leftarrow} \mathbb{A}^{0}$.

Associated to each straight-line program of length $s$, there is a morphism $\left(f_{1}, \ldots, f_{s}\right): \mathbb{A}^{n} \rightarrow \mathbb{A}^{s}$, where $f_{1}, \ldots, f_{s}$ are the polynomials computed in each line of the diagram. Let $\Gamma=\left(\Gamma_{1}, \ldots, \Gamma_{N}\right)$, where $\Gamma_{i}$ is the $i$ th instruction in the straight-line program $\Gamma$. For the first $n$ instructions of $\Gamma$, which introduce the variables $x_{1}, \ldots, x_{n}$ as polynomials, we have the map $\mathbb{A}^{n} \stackrel{\text { id }}{\rightarrow} \mathbb{A}^{n}$. The space $\mathbb{A}^{n}$ is constructed in a limit computation by taking the limit of $n$ disjoint copies of the diagram consisting of $\mathbb{A}^{1}$ mapping to itself by 1 . To get the map $\mathbb{A}^{n} \stackrel{\text { id }}{\rightarrow} \mathbb{A}^{n}$, we take the limit of the whole diagram obtained so far which $\operatorname{costs} n+2$. Note that our diagram also contains all the projections from the source and target of $\operatorname{id}_{\mathbb{A}^{n}}$ to the $n$ components. This is the base case of the following inductive construction.

Assume that, for $n \leqslant k \leqslant N-1$, we have produced, in a limit computation, a morphism $\left(f_{1}, \ldots, f_{k}\right): \mathbb{A}^{n} \rightarrow \mathbb{A}^{k}$ corresponding to the polynomials computed by each line of $\left(\Gamma_{1}, \ldots, \Gamma_{k}\right)$, together with the projections $p_{1}, \ldots, p_{k}$ from $\mathbb{A}^{k}$ to $k$ copies of $\mathbb{A}^{1}$, all of which are in $D_{0}$.

Assume that $\Gamma_{k+1}$ is the multiplication of the $i$ th and $j$ th lines of the program, that is, $f_{k+1}=f_{i} f_{j}$. We can then perform the following steps of the computation. For convenience, we have added, as if they were basic morphisms, the portion of the diagram used for the next steps as the first $k+1$ steps in this description:

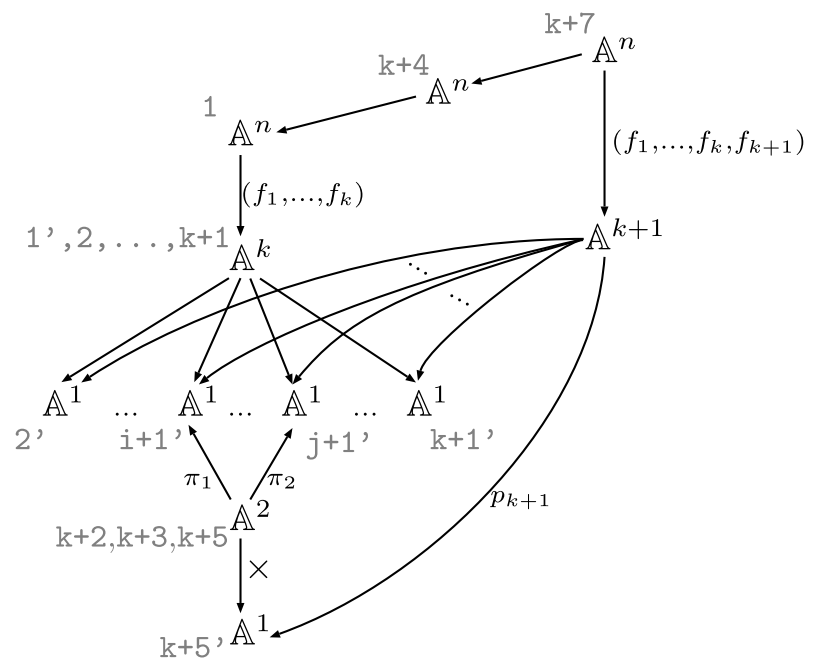




$$
\begin{aligned}
& \text { 1. } \quad, \mathbb{A}^{n} \stackrel{\left(f_{1}, \ldots, f_{k}\right)}{\longrightarrow} \mathbb{A}^{k}, 1^{\prime} \\
& \text { 2. } 1^{\prime}, \mathbb{A}^{k} \stackrel{p_{1}}{\rightarrow} \mathbb{A}^{1}, 2^{\prime} \\
& \text { 3. } 1^{\prime}, \mathbb{A}^{k} \stackrel{p_{2}}{\rightarrow} \mathbb{A}^{1}, 3^{\prime} \\
& \text {... } \\
& \mathrm{k}+1 . \quad 1^{\prime}, \mathbb{A}^{k} \stackrel{p_{k}}{\rightarrow} \mathbb{A}^{1},(\mathrm{k}+1)^{\prime} \\
& \mathrm{k}+2 . \quad-, \mathbb{A}^{2} \stackrel{\pi_{1}}{\rightarrow} \mathbb{A}^{1},(i+1)^{\prime} \\
& k+3 . \quad k+2, \mathbb{A}^{2} \stackrel{\pi_{2}}{\rightarrow} \mathbb{A}^{1},(j+1)^{\prime} \\
& k+4 \text {. } \lim \left((k+2),(i+1)^{\prime},(j+1)^{\prime}, 1^{\prime}, 1\right) \\
& \mathrm{k}+5 . \quad \mathrm{k}+2, \mathbb{A}^{2} \stackrel{\times}{\rightarrow} \mathbb{A}^{1}, \\
& \mathrm{k}+6 . \quad \lim \left(2^{\prime}, 3^{\prime}, \ldots,(\mathrm{k}+1)^{\prime},(\mathrm{k}+4)^{\prime}\right) \\
& k+7 . \quad \lim \left((k+4), 1,1^{\prime}, 2^{\prime}, 3^{\prime}, \ldots,(k+1)^{\prime}, k+2,(k+5)^{\prime}, k+6\right)
\end{aligned}
$$

In the end, the map $(k+7)->(k+6)$ is the map $\mathbb{A}^{n} \stackrel{\left(f_{1}, \ldots, f_{k+1}\right)}{\longrightarrow} \mathbb{A}^{k+1}$, and the projection maps for the next step are the maps

$$
(k+6)->2^{\prime},(k+6)->3^{\prime}, \ldots,(k+6)->(k+1)^{\prime},(k+6)->(k+5)^{\prime} .
$$

The process for addition steps is similar, with the step $k+5$ modified. For scalar multiplication, it is similar with two steps less. For constants appearing in the computation, we add a new variable and take the fiber product with $\mathbb{A}^{0} \stackrel{c}{\rightarrow} \mathbb{A}^{1}$ to fix the variable to the value $c$. Repeating this process until $k=N$, we see that $\left(f_{1}, \ldots, f_{N}\right)$ is produced in $\mathrm{O}(N)$ steps. We can then compose with the projection to the last coordinate, by taking a limit, to produce $\mathbb{A}^{n} \stackrel{f}{\rightarrow} \mathbb{A}^{1}$. To obtain the zeroset of $f$, we add $\mathbb{A}^{0} \stackrel{0}{\rightarrow} \mathbb{A}^{1}$ to this last $\mathbb{A}^{1}$ and take the limit.

It should also be noted that the way we made a limit computation for $\mathbb{A}^{n} \stackrel{f}{\rightarrow} \mathbb{A}^{1}$ was not the most efficient, but this way gives the cleanest inductive argument. For efficiency, the intermediate limit steps can be removed; cf. Lemma 3.19.

REMARK 4.3. The proof above shows that we can, by starting from a number of $\mathbb{A}^{1}$ added as basic objects, construct any polynomial functions $f_{1}: \mathbb{A}^{n} \rightarrow \mathbb{A}^{1}$. We will use this fact later.

We now consider a converse for Theorem 4.2 and show that, given a limit computation with low cost, an object $X$ computed by it is isomorphic to the zeroset of a polynomial whose arithmetic circuit complexity is low. Since diagram computations produce objects up to isomorphism, categorical complexity does not reflect the complexity of every polynomial that might be used to cut out $X$ in a larger space. For example, $X$ could be the graph of a polynomial map $f: \mathbb{A}^{n} \rightarrow \mathbb{A}^{1}$ with very high arithmetic circuit complexity, but since $X$ would be isomorphic to $\mathbb{A}^{n}$, its limit complexity would be very small, which does not say 
anything about the arithmetic circuit complexity of $f$. This is discussed in more detail in Section 5.1.

We first recall the definition of an arithmetic circuit over a field $k$ and that of the arithmetic circuit complexity of a polynomial map.

DEFINITION 4.4 (Arithmetic circuit complexity). An arithmetic circuit $C$ over a field $k$ and variables $X_{1}, \ldots, X_{n}$ is a finite directed acyclic graph such that

(i) every vertex of the directed graph with indegree 0 is labeled either by a variable or an element of $k$;

(ii) every other vertex is labeled by either + or $\times$.

The size of an arithmetic circuit is the number of vertices in the associated directed graph. Each vertex of $C$ is associated to a polynomial in $k\left[X_{1}, \ldots, X_{n}\right]$ computed at that vertex (in the obvious way). We say that a polynomial is computed by an arithmetic circuit $C$ if it appears as the polynomial associated to some vertex of the circuit. For a tuple of polynomials $f=\left(f_{1}, \ldots, f_{m}\right) \in k\left[X_{1}, \ldots, X_{n}\right]$, we say that the arithmetic circuit complexity of the induced polynomial map $f: k^{n} \rightarrow k^{m}$ is the size of the arithmetic circuit of the smallest size that computes each of the polynomials $f_{1}, \ldots, f_{m}$.

TheOREm 4.5. Let $\left(D_{0}, \ldots, D_{s}\right)$ be a limit computation in $\mathbf{A f f V a r}_{k}$, whose cost equals $C=c\left(D_{0}, \ldots, D_{s}\right)$. Then we have the following:

(i) If $X$ is an object computed by $\left(D_{0}, \ldots, D_{s}\right)$, then $X$ is isomorphic to the zero-set of a polynomial morphism $\mathbb{A}^{m_{1}} \rightarrow \mathbb{A}^{m_{2}}$ whose components are polynomials of degree at most 2 . The total arithmetic circuit complexity of the map is bounded above by $4 C$.

(ii) Every morphism $f: X \rightarrow Y$ in $D_{s}$ is the restriction of a projection $\mathbb{A}^{m_{1}} \rightarrow$ $\mathbb{A}^{m_{2}}$, where $\mathbb{A}^{m_{1}}$ and $\mathbb{A}^{m_{2}}$ are the spaces from Part $(i)$, where $X$ and $Y$ are embedded respectively.

Proof. If $s=0$, then the statement is true since the basic morphisms are $\mathbb{A}^{1} \stackrel{c}{\rightarrow} \mathbb{A}^{1}$, $\mathbb{A}^{1} \times \mathbb{A}^{1} \stackrel{+, \times}{\longrightarrow} \mathbb{A}^{1}, \mathbb{A}^{1} \times \mathbb{A}^{1} \stackrel{\pi_{1}, \pi_{2}}{\rightarrow} \mathbb{A}^{1}, \mathbb{A}^{1} \rightarrow \mathbb{A}^{0}$, and $\mathbb{A}^{0} \stackrel{c}{\rightarrow} \mathbb{A}^{1}$.

For the general case, constructivity implies that, as far as the isomorphism class of an object $X$ in $D_{s}$ is concerned, the intermediate limits in a limit computation can be removed (Lemma 3.19). Using the notation of the proof of Lemma 3.19, if $X=L_{i}$ is produced as a limit in the diagram computation, then $\left.X \cong \lim D_{i-1}\right|_{J_{i}^{\prime}}$, where $\left.D_{i-1}\right|_{J^{\prime}}$ is a subdiagram of $D_{0}$. 
As discussed in Remark 3.20, we can write $X=L_{i}$ as an equalizer. Let $J_{i}^{\prime}=$ $(V, E),\left.L_{i} \cong \lim D_{i-1}\right|_{J_{i}^{\prime}}$; writing $D=\left.D_{i-1}\right|_{J_{i}^{\prime}}, L_{i}$ is isomorphic to the limit of

$$
\prod_{v \in V} D(v) \underset{\phi}{\stackrel{\psi}{\longrightarrow}} \prod_{e \in E} D(t(e)),
$$

where $\phi_{D(t(e))}=\pi_{D(t(e))}$ and $\psi_{D(t(e))}=D(e) \circ \pi_{D(s(e))}$.

Since $J_{i}^{\prime} \subset I_{0}$, we have that each $D(v)$ or $D(t(e))$ is $\mathbb{A}^{1}, \mathbb{A}^{2}$, or a point; and $D(e)$ are addition, multiplication, constant, projection, or multiplication by a constant. So the above equalizer is of the form

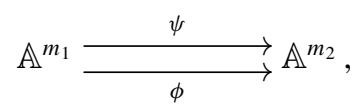

and $L_{i}$ is isomorphic to the zero-locus of $\mathbb{A}^{m_{1}} \stackrel{\phi-\psi}{\longrightarrow} \mathbb{A}^{m_{2}}$. For the complexity of $\phi-\psi$, observe that $m_{1}, m_{2} \leqslant 2 C$ and that each component of $\phi-\psi$ is a very simple polynomial, which can be produced in two steps.

We now consider the second assertion. This follows directly from the discussion in Remark 3.21. We observe that any morphism $f: X \rightarrow Y$ appearing in $D_{s}$ but not in $D_{0}$ must be a cone map from a limit $X=L_{i}=\left.\lim D_{i-1}\right|_{J_{i}}$ to an object $Y$ appearing in $\left.D_{i-1}\right|_{J_{i}}$. By the above construction, we know that $X$ and $Y$ are zero-loci of morphisms whose sources are products of objects in subdiagrams of $D_{0}$. Moreover, constructivity implies that the subdiagram for $Y$ is contained in the subdiagram for $X$. The morphism $f$ is then the restriction of the projection from the subdiagram for $X$ to the subdiagram for $Y$.

The above arguments can also be considered for the category of affine schemes instead of varieties. Let $k$ be a field and let $\mathbf{A f f S c h}$ be the category of affine schemes. Consider the same set $\mathcal{A}$ of morphisms as in the case of affine varieties: $\mathbb{A}^{1} \stackrel{c}{\rightarrow} \mathbb{A}^{1}$, for each $c \in k, \mathbb{A}^{1} \times \mathbb{A}^{1} \stackrel{+}{\rightarrow} \mathbb{A}^{1}, \mathbb{A}^{1} \times \mathbb{A}^{1} \stackrel{\times}{\rightarrow} \mathbb{A}^{1}, \mathbb{A}^{1} \times \mathbb{A}^{1} \stackrel{\pi_{1}, \pi_{2}}{\rightarrow} \mathbb{A}^{1}$, $\mathbb{A}^{1} \rightarrow \mathbb{A}^{0}$, and $\mathbb{A}^{0} \stackrel{c}{\rightarrow} \mathbb{A}^{1}$.

One can also consider the category $\mathbf{A l g}_{k}$ of $k$-algebras with basic morphisms

$$
\begin{aligned}
& k[x] \stackrel{c}{\rightarrow} k[x], \quad \text { for each } c \in k, \\
& k[x] \stackrel{x \otimes 1+1 \otimes x}{\longrightarrow} k[x] \otimes k[x], \\
& k[x] \stackrel{x \otimes x}{\longrightarrow} k[x] \otimes k[x], \\
& k[x] \stackrel{\text { id } \otimes 1,1 \otimes \text { id }}{\longrightarrow} k[x] \otimes k[x], \\
& k \stackrel{1}{\rightarrow} k[x], \quad \text { and } \\
& k[x] \stackrel{x \mapsto c}{\longrightarrow} k, \quad \text { for each } c \in k .
\end{aligned}
$$


These correspond to the basic morphisms considered for $\mathbf{A f f}_{\mathbf{V a r}}$ under the adjoint equivalence

$$
\operatorname{AffSch}_{k} \underset{\text { Spec }}{\stackrel{k[\cdot]}{\longrightarrow}} \mathbf{A l g}_{k}^{\text {op }} .
$$

Without any modification to the proofs, Theorems 4.2 and 4.5 hold when $\mathbf{A f f V a r}_{k}$ is replaced by $\mathbf{A f f S c h}$ or $\mathbf{A l g}_{k}$.

4.2. Mixed computations in projective schemes. Let $\mathcal{C}$ be the category $\operatorname{Sch}_{k}$ of all schemes over a field $k$. Letting $\mathcal{A}$ consist of the morphisms above as in the affine scheme case, we get a definition of complexity in the category of schemes.

The morphisms in $\mathcal{A}$ are actually enough to produce all projective schemes using mixed computations. For example, in order to make $\mathbb{P}^{1}$, we can produce the following diagram as a subdiagram of a computation and take its colimit:

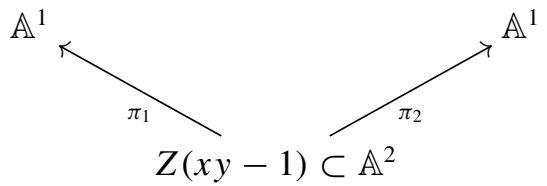

Note that $Z(x y-1)$ is isomorphic to $\mathbb{A}^{1}-\{0\}$.

Proposition 4.6. Let $X \subset \mathbb{P}_{k}^{n}$ be the zero-scheme of homogeneous polynomials $f_{1}, \ldots, f_{m} \in k\left[x_{0}, \ldots, x_{n}\right]$. Assume that, for each $i$, there is an arithmetic circuit of size $c_{i}$ computing $f_{i}$. Then, the categorical complexity of $X$ is in $\mathrm{O}\left(n^{2}\left(c_{1}+\right.\right.$ $\left.\cdots+c_{s}\right)$ ).

Proof. We first show how to construct $\mathbb{P}^{n}$ by a mixed computation and then modify the construction to make the zero-scheme $X$ of $f_{1}, \ldots, f_{m}$.

The last step in making $\mathbb{P}^{n}$ will be to take the colimit of the diagram

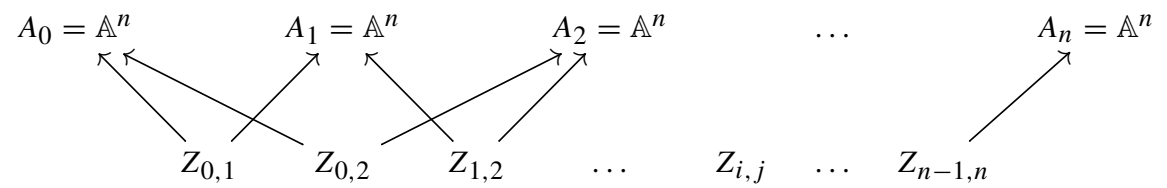

There are $n+1$ copies of $\mathbb{A}^{n}$ in the first row, which correspond to the standard covering $U_{0}, \ldots, U_{n}$ of $\mathbb{P}^{n}$ by affine opens. In the second row, there is an object $Z_{i, j}$ for each pair $(i, j)$ with $i<j$; with each $Z_{i, j}$ corresponding to the 
intersections $U_{i} \cap U_{j}$ of the affine opens in the chart, each is therefore isomorphic to the complement of a hyperplane in $\mathbb{A}^{n}$.

We will construct each $Z_{i j}$ as a subscheme of $\mathbb{A}^{n} \times \mathbb{A}^{n}=A_{i} \times A_{j}$, considered with coordinates $x_{1}, \ldots, x_{n}, y_{1}, \ldots, y_{n}$, defined by the equation

$$
y_{i} x_{j}-1=0,
$$

and, for each $l \in\{1, \ldots, i-1, i+1, \ldots, n\}$, the equation

$$
y_{i} x_{l}-y_{l}=0 .
$$

These equations describe the graph of the transition maps

$$
\left(x_{1}, \ldots, x_{n}\right) \mapsto\left(\frac{x_{1}}{x_{j}}, \frac{x_{2}}{x_{j}}, \ldots, \frac{x_{i-1}}{x_{j}}, \frac{1}{x_{j}}, \frac{x_{i+1}}{x_{j}}, \ldots, \frac{x_{j-1}}{x_{j}}, \frac{x_{j+1}}{x_{j}}, \ldots, \frac{x_{n}}{x_{j}}\right)
$$

between the affine opens in the standard covering of $\mathbb{P}^{n}$. Here, each affine open $U_{i}$, corresponding to points in homogeneous coordinates $\left[\frac{a_{0}}{a_{i}}: \cdots: \frac{a_{i-1}}{a_{i}}: 1: \frac{a_{i+1}}{a_{i}}: \cdots\right.$ : $\left.\frac{a_{n}}{a_{i}}\right]$ is parameterized by simply omitting the $i$ th variable. The maps $Z_{i, j} \rightarrow A_{i}$ and $Z_{i, j} \rightarrow A_{j}$ are the restrictions of the projection maps from $A_{i} \times A_{j}$.

To carry out the computation, start with $(n+1)$ sets of $n$ copies of $\mathbb{A}^{1}$. Make $A_{i}$ the limit of the $i$ th set of $n \mathbb{A}^{1}$ 's. Using the procedure described in the proof of Theorem 4.2 (cf. Remark 4.3), construct each $Z_{i, j}$ as the zero-set of equations (5) and (6). Making the projections to the $A_{i}$ (cf. second part of Remark 3.8), we get the diagram above. At this point, the colimit of this diagram can be taken to produce $\mathbb{P}^{n}$.

To make the zero-scheme $X$ of $f_{1}, \ldots, f_{m}$ in $\mathbb{P}^{n}$, we continue in order to make the following diagram.

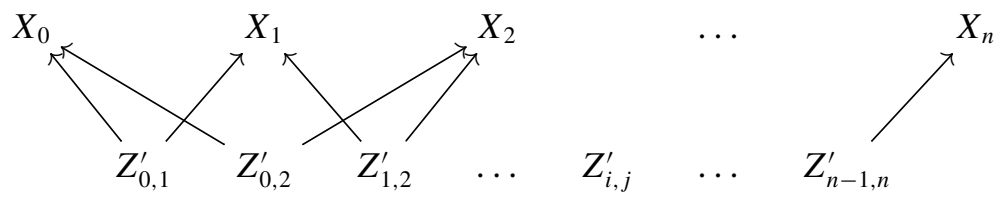

Here the $X_{i}$ are isomorphic to $X \cap U_{i}$ and the $Z_{i, j}^{\prime}$ are isomorphic to $X \cap U_{i} \cap U_{j}$. To make the $X_{i}$, de-homogenize $f_{1}, \ldots, f_{m}$ for each $A_{i}$ so that we get the equations for the subscheme of $A_{i}$ that corresponds to $X \cap U_{i}$. Using the procedure in Theorem 4.2, take the zero-scheme of these de-homogenized equations in each $A_{i}$. To make the $Z_{i, j}^{\prime}$, make the map $X_{i} \times X_{j} \rightarrow A_{i} \times A_{j}$ and pull back $Z_{i, j}$. Finally, take the colimit of the diagram above to get a scheme isomorphic to $X$. 


\section{Categorical complexity of morphisms versus circuit complexity of polynomials}

Although the motivation behind studying categorical complexity in various categories is a natural problem in its own right, it is still interesting to compare this new notion with pre-existing notions of computational complexity in certain special categories. For a pre-existing notion of complexity, the closest in spirit (in the categories we consider below) is that of arithmetic circuit complexity of polynomials. The goal of this section is to see how closely we can recover arithmetic circuit complexity (defined in Definition 4.4) (or equivalently, straightline program complexity). We remark that arithmetic circuit complexity is one of the most widely studied frameworks for nonuniform computational complexity theory (see for example, [Bür00]).

We discuss three categories where we can compare the arithmetic circuit complexity of a polynomial $f \in k\left[x_{1}, \ldots, x_{n}\right]$ with the categorical complexity of a morphism diagram. In the first one, we look at the morphism diagram $\mathbf{D}_{f}=\left(\mathbb{A}^{n} \stackrel{f}{\rightarrow} \mathbb{A}^{1}\right)$ in $\mathbf{A f f} \mathbf{V a r}_{k}$ and see that its complexity can be very different from the arithmetic circuit complexity of $f$. The second category, the category of graded pairs of algebras, is an attempt at removing this discrepancy. The third category is the category of modules over $k\left[x_{1}, \ldots, x_{n}\right]$, where we prove concrete comparison results between the arithmetic circuit complexity of $f$ and the categorical complexity of the morphism diagram $k\left[x_{1}, \ldots, x_{n}\right] \stackrel{1 \mapsto f}{\longrightarrow} k\left[x_{1}\right.$, $\left.\ldots, x_{n}\right]$.

5.1. Complexity of polynomial morphisms in $\operatorname{AffVar}_{k}$. We consider the category $\mathbf{A f f V a r}_{k}$ with the basic morphisms discussed above (4). Given a polynomial

$$
f \in k\left[x_{1}, \ldots, x_{n}\right],
$$

what is the categorical complexity of the diagram $\mathrm{D}_{f}=\left(\mathbb{A}^{n} \stackrel{f}{\rightarrow} \mathbb{A}^{1}\right)$ in $\mathbf{A f f V a r}_{k}$ ?

First, observe that since we have $\mathbb{A}^{0} \stackrel{0}{\rightarrow} \mathbb{A}^{1}$ as a basic morphism, we can use the pullback of the diagram

$$
\mathbb{A}^{n} \stackrel{f}{\longrightarrow} \mathbb{A}^{1} \stackrel{0}{\longleftarrow} \mathbb{A}^{0}
$$

to obtain the inclusion morphism $V \hookrightarrow \mathbb{A}^{n}$, where $V=Z(f)$ is the affine subvariety of $\mathbb{A}^{n}$ defined by $f$. Thus,

$$
c_{\mathbf{A f f V a r}_{k}, \mathcal{A}}^{\lim }(Z(f)) \leqslant c_{\mathbf{A f f V a r}_{k}, \mathcal{A}}^{\lim }\left(\mathrm{D}_{f}\right)+2,
$$

where $\mathcal{A}$ is the set of basic morphisms given in (4). 
Categorical computations produce diagrams up to isomorphism, and categorical complexity is defined for isomorphism classes of diagrams. So, the complexity of $f$ is equal to the complexity of any other $\mathbb{A}^{n} \stackrel{g}{\rightarrow} \mathbb{A}^{1}$ where there is an automorphism $\phi: \mathbb{A}^{n} \rightarrow \mathbb{A}^{n}$ such that

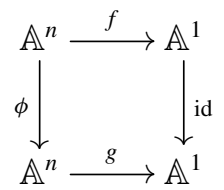

commutes. Therefore, the complexity of $\mathrm{D}_{f}$ in $\mathbf{A f f V a r}_{k}$ is invariant under polynomial automorphisms of $\mathbb{A}^{n}$ (and the complexity of the variety $Z(f)$ is equal to that of $\phi(Z(f))$ for any polynomial automorphism $\phi$ of $\left.\mathbb{A}^{n}\right)$.

For example, let $p \in k\left[x_{1}, \ldots, x_{n}\right]$ be any polynomial, and let

$$
g \in k\left[x_{1}, \ldots, x_{n}, x_{n+1}\right], \quad g=x_{n+1}+p\left(x_{1}, \ldots, x_{n}\right) .
$$

Let $f=\pi_{n+1}$ sending $\left(x_{1}, \ldots, x_{n+1}\right)$ to $x_{n+1}$, and

$$
\phi\left(x_{1}, \ldots, x_{n+1}\right)=\left(x_{1}, \ldots, x_{n}, x_{n+1}+p\left(x_{1}, \ldots, x_{n}\right)\right) .
$$

Then, we have the following commuting diagram:

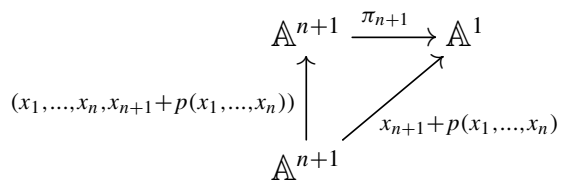

So, the diagram $\mathrm{D}_{x_{n+1}+p\left(x_{1}, \ldots, x_{n}\right)}$ is isomorphic to $\mathbb{A}^{n+1} \stackrel{\pi_{n+1}}{\longrightarrow} \mathbb{A}^{1}$. So, while the circuit complexity of $x_{n+1}+p\left(x_{1}, \ldots, x_{n}\right)$ can be very high (for example, $p$ could be the permanent, or worse, a generic polynomial), the limit complexity of $\mathrm{D}_{x_{n+1}+p\left(x_{1}, \ldots, x_{n}\right)}$ as well as that of $V\left(x_{n+1}+p\left(x_{1}, \ldots, x_{n}\right)\right)$ is bounded by $O(n)$. This is because, geometrically, the zero-set of $x_{n+1}+p\left(x_{1}, \ldots, x_{n}\right)$ is very simple; it is the graph of $-p$, and is therefore isomorphic to $\mathbb{A}^{n}$.

It should also be noted that reductions in circuit complexity do not immediately lead to reductions in the complexity in $\mathbf{A f f V a r}_{k}$. One can construct an arithmetic circuit for the polynomial $p\left(x_{1}, \ldots, x_{n}\right)$ given one for $x_{n+1}+p\left(x_{1}, \ldots, x_{n}\right)$ with only a constant increase in size. However, this does not lead to a diagram computation of $D_{p\left(x_{1}, \ldots, x_{n}\right)}$ from a diagram computation of $D_{x_{n+1}+p\left(x_{1}, \ldots, x_{n}\right)}$ with only a constant increase in cost. Still, we can ask whether polynomials that are believed to be hard to compute in the arithmetic circuit model also have high categorical complexity. For example, we have the following question. 
QUESTION 5.1. Is the limit/mixed complexity of $\mathbb{A}^{n^{2}} \stackrel{\text { perm }_{n}}{\longrightarrow} \mathbb{A}^{1}$ polynomially bounded in $n$ ?

5.2. Complexity of pairs of graded algebras. It is possible that the difference between complexity of $\mathrm{D}_{f}$ in $\mathbf{A f f V a r}_{k}$ and the arithmetic circuit complexity of $f$ is caused by the large number of automorphisms of $\mathbb{A}^{n}$. The goal of this section is to consider a category where arithmetic circuit complexity is possibly close to categorical complexity, and we can hope to bound the arithmetic circuit complexity of a polynomial $f$ from above by a polynomial function of the categorical complexity of the corresponding morphism (or that of the algebraic variety defined by $f$ ), and thus match the result in Part (i) of Theorem 4.5 , which contains an inequality in the other direction.

The automorphism group of $\mathbb{P}_{k}^{n}$ is much smaller than that of $\mathbb{A}_{k}^{n}$. Indeed, $\operatorname{Aut}\left(\mathbb{P}_{k}^{n}\right) \cong P G L(n+1, k)$, where $P G L(n+1, k)$ denotes the group of invertible $(n+1) \times(n+1)$ matrices with entries in $k$ modulo multiplication by $k^{*}$ (see for example, [MFK94, Ch. 0, $\$ 5$ b)]). Thus, the automorphisms of $\mathbb{P}_{k}^{n}$ are all linear, and hence the complexity of a projective hypersurface $Z(f) \subset \mathbb{P}_{k}^{N}$ should be closer to the arithmetic circuit complexity of its defining polynomial $f$. However, since the basic objects are not projective, it is better to consider the corresponding setup in the category of graded algebras.

To this end, we could consider the category $\mathbf{G r A l g}_{k}$ of graded algebras (given a graded $k$-algebra $S$, the projective scheme Proj $S$ will be the corresponding geometric object). The following set of morphisms will be taken to be the basic morphisms.

$$
\begin{aligned}
k\left[z^{n}\right] \stackrel{+}{\rightarrow} k\left[x^{n}, y^{n}\right], z^{n} \mapsto x^{n}+y^{n}, \\
k\left[z^{2 n}\right] \stackrel{\dot{\rightarrow}}{\rightarrow} k\left[x^{n}, y^{n}\right], z^{2 n} \mapsto x^{n} y^{n}, \\
k\left[z^{n}\right] \stackrel{i_{1}}{\rightarrow} k\left[x^{n}, y^{n}\right], z^{n} \mapsto x^{n}, \\
k\left[z^{n}\right] \stackrel{i_{2}}{\rightarrow} k\left[x^{n}, y^{n}\right], z^{n} \mapsto y^{n}, \\
k\left[z^{n}\right] \stackrel{c x}{\rightarrow} k\left[z^{n}\right], z^{n} \mapsto c z^{n}, c \in k, \\
k\left[z^{n}\right] \rightarrow k, z^{n} \mapsto 0 .
\end{aligned}
$$

Note that $k\left[x^{n}, y^{n}\right] \cong k\left[x^{n}\right] \otimes_{k} k\left[y^{n}\right]$ is the coproduct of $k\left[x^{n}\right]$ and $k\left[y^{n}\right]$ in $\mathbf{G r A l g}_{k}$, and hence using colimits we can build graded polynomial rings in any number of variables. Also, the last morphism in list (7) allows us, 'geometrically speaking', to build the inclusion of a variety $V \hookrightarrow \mathbb{P}_{k}^{N}$ defined by a set of homogeneous polynomials of the same degree. More precisely, in terms of graded algebras, if $V$ is defined by the ideal $\mathfrak{a}$ with generators $g_{1}, \ldots, g_{M} \in k\left[z_{0}, \ldots, z_{N}\right]$ 
homogeneous of the same degree, then the morphism $k\left[z_{0}, \ldots, z_{N}\right] \rightarrow k\left[z_{0}, \ldots\right.$, $\left.z_{N}\right] / \mathfrak{a}$ can be constructed by taking the colimit of the diagram

$$
k \stackrel{0}{\longleftarrow} k\left[y_{0}, \ldots, y_{M}\right] \stackrel{y_{i} \mapsto g_{i}}{\longrightarrow} k\left[z_{0}, \ldots z_{N}\right],
$$

noting that the morphism $k\left[y_{0}, \ldots, y_{M}\right] \stackrel{0}{\rightarrow} k$ can be built from the basic morphism $k[z] \rightarrow k, z \mapsto 0$ (cf. equation (7)) by taking colimits.

REMARK 5.2. We should remind the reader here of one unpleasant aspect of the translation between algebra and geometry via taking Proj of graded rings. Unlike the functor

$$
\text { Spec }: \mathbf{A l g}_{k}^{\text {op }} \rightarrow \mathbf{A f f S c h}_{k}
$$

the map

$$
\text { Proj : } \text { GrAlg }_{k}^{\text {op }} \rightarrow \text { ProjSch }_{k}
$$

is not a functor since to a morphism of graded $k$-algebras one can associate only a partial map between the corresponding projective schemes, and for graded $k$-algebras $R$ and $R^{\prime}$, Proj $R$ and Proj $R^{\prime}$ could be isomorphic, without $R$ being isomorphic to $R^{\prime}$. Thus, isomorphisms of projective varieties considered in this section do not necessarily correspond exactly to isomorphisms of the corresponding graded algebras. It might be possible to consider as our objects equivalence classes of graded algebras $S$ giving isomorphic Proj $S$, but we will not attempt to do this here.

Now consider for a tuple of homogeneous polynomial

$$
f=\left(f_{0}, \ldots, f_{N}\right) \in k\left[x_{0}, \ldots, x_{n}\right]^{N+1},
$$

the diagram

$$
k\left[z_{0}, \ldots, z_{N}\right] \stackrel{z_{i} \mapsto f_{i}}{\longrightarrow} k\left[x_{0}, \ldots, x_{n}\right]
$$

denoted also by $\mathrm{D}_{f}$.

However, the complexity of $\mathrm{D}_{f}$ could still possibly be different from the arithmetic circuit complexity of $f$. The problem arises if morphism $\mathbb{P}_{k}^{n} \rightarrow \mathbb{P}_{k}^{N}$ induced by $f$ is an embedding of varieties. More precisely, an embedding $v: \mathbb{P}_{k}^{n}=\operatorname{Proj} k\left[x_{0}, \ldots, x_{n}\right] \rightarrow \mathbb{P}_{k}^{N}=\operatorname{Proj} k\left[z_{0}, \ldots, z_{N}\right]$. In terms of graded algebras, this would correspond to a morphism $\mathrm{D}_{f}: k\left[z_{0}, \ldots, z_{N}\right] \rightarrow k\left[x_{0}\right.$, $\left.\ldots, x_{n}\right]$ given by a tuple $f=\left(f_{0}, \ldots, f_{N}\right)$ of homogeneous polynomials of the same degrees, with $\mathrm{D}_{f}$ being the map $z_{i} \mapsto f_{i}$. For example, $v$ could be the Veronese embedding of degree $d$, and in which case $N=\left(\begin{array}{c}n+d \\ d\end{array}\right)$, and $f$ 
would be the tuple of all monomials in $x_{0}, \ldots, x_{n}$ of degree $d$. But the inclusion $v\left(\mathbb{P}_{k}^{n}\right) \hookrightarrow \mathbb{P}_{k}^{N}$ also corresponds to a morphism of graded algebras, namely $k\left[z_{0}\right.$, $\left.\ldots, z_{N}\right] \rightarrow k\left[z_{0}, \ldots, z_{N}\right] / \mathfrak{a}$, where $\mathfrak{a}$ is the homogeneous ideal of $v\left(\mathbb{P}_{k}^{n}\right)$. Suppose that $\mathfrak{a}$ is generated by the homogeneous polynomials $g_{0}, \ldots, g_{M}$. In the case where $v$ is a Veronese embedding, the polynomials $g_{i}$ can be taken to be a set of quadratic binomials. Now the morphism $k\left[z_{0}, \ldots, z_{N}\right] \rightarrow k\left[z_{0}, \ldots, z_{N}\right] / \mathfrak{a}$ can be constructed by taking the colimit (see (8) above).

If the $g_{i}$ 's have small colimit complexity compared to the $f_{i}$ 's, then the second morphism will have smaller colimit complexity. And thus the categorical complexity of the embedding $v: \mathbb{P}_{k}^{n} \rightarrow \mathbb{P}_{k}^{N}$ could be determined by a categorical computation involving only the polynomials $g_{0}, \ldots, g_{M}$, and in principle could be much smaller than the arithmetic circuit complexity of $f$.

To prevent the phenomenon described above, which might cause the categorical complexity of a morphism $\mathrm{D}_{f}$ to diverge from the arithmetic circuit complexity of $f$, we consider the category whose objects are pairs $\left(X, \mathbb{P}_{k}^{n}\right)$, with $X$ a subvariety of $\mathbb{P}_{k}^{n}$. Or rather, we consider the corresponding morphisms of the coordinate algebras. We denote by GrAlgPairs , $_{k}$ the category whose objects are surjective morphisms $\left(A \stackrel{f}{\rightarrow} B\right.$ ), where $A$ is isomorphic to some polynomial ring $k\left[x_{0}, \ldots\right.$, $\left.x_{n}\right]$ graded by degree. For example, suppose that $A=k[x]$ and $B=k[x] /\left(x^{2}\right)$ both graded by degree, and $A \stackrel{f}{\rightarrow} B$ the canonical surjection.

In order to define categorical complexity in GrAlgPairs $\mathbf{G}_{k}$, we define the basic morphisms as follows. For each $n \geqslant 1$, we include in the set of basic morphisms the following set of morphisms (all polynomial rings appearing below are graded by degrees and the degrees of the indeterminates $x, y, z, \ldots$ appearing in the morphisms are all equal to 1 ):

$$
\begin{aligned}
&\left(k\left[z^{n}\right] \stackrel{\sim}{\rightarrow} k\left[z^{n}\right]\right) \stackrel{+}{\rightarrow}\left(k\left[x^{n}, y^{n}\right] \stackrel{\sim}{\rightarrow} k\left[x^{n}, y^{n}\right]\right), z^{n} \mapsto x^{n}+y^{n}, \\
&\left(k\left[z^{2 n}\right] \stackrel{\sim}{\rightarrow} k\left[z^{2 n}\right]\right) \stackrel{\rightarrow}{\rightarrow}\left(k\left[x^{n}, y^{n}\right] \stackrel{\stackrel{\sim}{\rightarrow}}{ } k\left[x^{n}, y^{n}\right]\right), z^{2 n} \mapsto x^{n} y^{n}, \\
&\left(k\left[z^{n}\right] \stackrel{\sim}{\rightarrow} k\left[z^{n}\right]\right) \stackrel{i_{1}}{\rightarrow}\left(k\left[x^{n}, y^{n}\right] \stackrel{\sim}{\rightarrow} k\left[x^{n}, y^{n}\right]\right), z^{n} \mapsto x^{n}, \\
&\left(k\left[z^{n}\right] \stackrel{\sim}{\rightarrow} k\left[z^{n}\right]\right) \stackrel{\sim}{\rightarrow}\left(k\left[x^{n}, y^{n}\right] \stackrel{\sim}{\rightarrow} k\left[x^{n}, y^{n}\right]\right), z^{n} \mapsto y^{n}, \\
&\left(k\left[z^{n}\right] \stackrel{\sim}{\rightarrow} k\left[z^{n}\right]\right) \stackrel{c \times}{\rightarrow}\left(k\left[z^{n}\right] \stackrel{\sim}{\rightarrow} k\left[z^{n}\right]\right), z^{n} \mapsto c z^{n}, c \in k, \\
&\left(k\left[z^{n}\right] \stackrel{\sim}{\rightarrow} k\left[z^{n}\right]\right) \rightarrow\left(k\left[x^{n}\right] \stackrel{(x \mapsto 0)}{\rightarrow} k\right),\left(z^{n} \mapsto x^{n}, z^{n} \mapsto 0\right) .
\end{aligned}
$$

REMARK 5.3. Note that in all but the last morphism in the above list, the pairs occurring as source and target of the morphism consist of isomorphic objects, and the morphism between them is diagonal (that is, of the form $(\phi, \phi)$ for some morphism $\phi$ in the category $\mathbf{G r A l g}_{k}$ ). Only in the last morphism this is not true, and the morphism between the pair in this case is (id, $\phi$ ), where $\phi$ is precisely the 
morphism used to build inclusion of varieties $\mathbf{G r A l g}_{k}$ (cf. (8)). This remark will be used in what follows.

Note that we can obtain the morphism

$$
\left(k\left[z^{n}\right] \stackrel{\left(z^{n} \mapsto 0\right)}{\longrightarrow} k\right) \stackrel{\left(z^{n} \mapsto 0\right)}{\longrightarrow}\left(k\left[x^{n}\right] \stackrel{\sim}{\rightarrow} k\left[x^{n}\right]\right)
$$

as the colimit diagram:

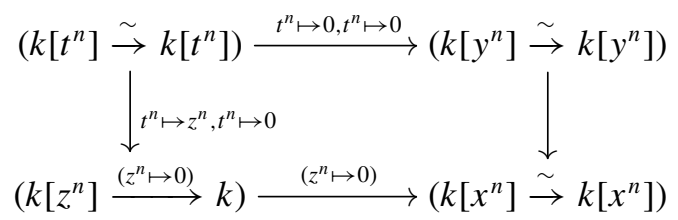

Given a tuple of homogeneous polynomials

$$
f=\left(f_{0}, \ldots, f_{N}\right) \in k\left[x_{0}, \ldots, x_{n}\right]^{N+1},
$$

we consider the categorical complexity of the diagram $\mathbf{M}_{f}$ defined as

$$
\left(k\left[z_{0}, \ldots, z_{N}\right] \stackrel{\sim}{\rightarrow} k\left[z_{0}, \ldots, z_{N}\right]\right) \stackrel{\mathrm{M}_{f}}{\longrightarrow}\left(k\left[z_{0}, \ldots, z_{n}\right] \stackrel{\sim}{\rightarrow} k\left[x_{0}, \ldots, x_{n}\right]\right), z_{i} \mapsto f_{i} .
$$

Following the same proof as Theorem 4.2, we have the next proposition.

Proposition 5.4. Given a tuple of homogeneous polynomials

$$
f=\left(f_{0}, \ldots, f_{N}\right) \in k\left[x_{0}, \ldots, x_{n}\right]^{N+1}
$$

having arithmetic circuit complexity $M$, the colimit complexity of the morphism diagram $\mathrm{M}_{f}$ is in $\mathrm{O}(M)$.

We claim also that the phenomenon described previously in the nonembedded setting does not occur in this embedded pair setting. Consider, for example, an embedding $v: \mathbb{P}_{k}^{n} \rightarrow \mathbb{P}_{k}^{N}$ as before, but now consider the embedding of the pair $\left(\mathbb{P}_{k}^{n}, \mathbb{P}_{k}^{n}\right)$ into $\left.\mathbb{P}_{k}^{N}, \mathbb{P}_{k}^{N}\right)$ by the diagonal morphism $(v, v)$. In terms of pairs of graded algebras, this would correspond to a morphism

$$
\begin{gathered}
\mathrm{M}_{f}=\left(\mathrm{D}_{f}, \mathrm{D}_{f}\right): \\
\left(k\left[z_{0}, \ldots, z_{N}\right] \stackrel{\sim}{\rightarrow} k\left[z_{0}, \ldots, z_{N}\right]\right) \stackrel{\rightarrow}{\rightarrow}\left(k\left[x_{0}, \ldots, x_{n}\right] \stackrel{\sim}{\rightarrow} k\left[x_{0}, \ldots, x_{n}\right]\right),
\end{gathered}
$$


given by a tuple $f=\left(f_{0}, \ldots, f_{N}\right)$ of homogeneous polynomials of the same degrees, with $\mathrm{D}_{f}$ being the map $z_{i} \mapsto f_{i}$. Suppose also as before that the inclusion $v\left(\mathbb{P}_{k}^{n}\right) \hookrightarrow \mathbb{P}_{k}^{N}$ also corresponds to a morphism of graded algebras, namely $k\left[z_{0}\right.$, $\left.\ldots, z_{N}\right] \rightarrow k\left[z_{0}, \ldots, z_{N}\right] / \mathfrak{a}$, where $\mathfrak{a}$ is the homogeneous ideal of $v\left(\mathbb{P}_{k}^{n}\right)$. Suppose that $\mathfrak{a}$ is generated by the homogeneous polynomials $g_{0}, \ldots, g_{M}$. However, unlike in the nonembedded case, we cannot construct the morphism

$$
\left(k\left[z_{0}, \ldots, z_{N}\right] \stackrel{\sim}{\rightarrow} k\left[z_{0}, \ldots, z_{N}\right]\right) \rightarrow\left(k\left[z_{0}, \ldots, z_{N}\right] / \mathfrak{a} \stackrel{\sim}{\rightarrow} k\left[z_{0}, \ldots, z_{N}\right] / \mathfrak{a}\right)
$$

using the polynomials $g_{i}$ and taking a colimit, since the morphism $k[z] \stackrel{0}{\rightarrow} k$ used to construct the morphism $k\left[y_{0}, \ldots, y_{M}\right] \stackrel{0}{\rightarrow} k$ in the colimit in (8) is available only in the second slot of the pairs and not in the first (see Remark 5.3).

We can now ask whether the converse of Proposition 5.4 is true.

QUESTION 5.5. For a tuple of homogeneous polynomials $f=\left(f_{0}, \ldots, f_{N}\right) \in$ $k\left[x_{0}, \ldots, x_{n}\right]^{N+1}$ for which the colimit complexity of the morphism $\mathbf{M}_{f}$ in GrAlgPairs $_{k}$ is $M$, is the arithmetic circuit complexity of $f$ polynomially bounded in $M$ ?

\subsection{Arithmetic circuit and categorical complexity of modules over} polynomial rings. We now consider the relationship between arithmetic circuit complexity and categorical complexity in categories of modules. Unlike in the categories of affine varieties and graded algebras (namely, $\mathbf{A f f} \mathbf{f}_{k}, \mathbf{G r A l g}$, GrAlgPairs $_{k}$ ) considered in the last two sections, we are able to relate polynomially the arithmetic circuit complexity and categorical complexity of polynomials (and their induced morphisms in the module category) by proving inequalities in both directions. Thus, we are able to prove a stronger relation between circuit complexity and categorical complexity-though the category is perhaps less interesting from the geometric point of view than the ones considered previously.

Let $R=k\left[x_{1}, \ldots, x_{n}\right]$. We consider the category $R$-Mod of $R$-modules. We consider colimit computations in $R$-Mod with the following set of basic morphisms.

$$
\begin{aligned}
& R \stackrel{x_{i}}{\rightarrow} R, \quad \text { for each } i=1, \ldots, n, \\
& R \stackrel{c}{\rightarrow} R, \quad \text { for each } c \in k, \\
& R \stackrel{i_{1}, i_{2}}{\rightarrow} R \oplus R, \\
& R \stackrel{\Delta}{\rightarrow} R \oplus R,
\end{aligned}
$$




$$
\begin{aligned}
& R \oplus R \stackrel{+}{\rightarrow} R, \\
& R \rightarrow\{0\} .
\end{aligned}
$$

For a polynomial $f \in k\left[x_{1}, \ldots, x_{n}\right]$, we consider the corresponding morphism $R \stackrel{f}{\rightarrow} R$ that sends 1 to $f$. Recall that a formula is an arithmetic circuit or straight-line program where past intermediate computations cannot be reused (see [Bür00, §2.2.1]).

PROPOSITION 5.6. If a polynomial $f \in k\left[x_{1}, \ldots, x_{n}\right]$ is computed by a formula of size $s$, then the diagram $R \stackrel{f}{\rightarrow} R$ is computed by a colimit computation in $R$-Mod with cost bounded by $\mathrm{O}(s)$.

Proof. Without loss of generality, assume that all sum and product gates have fanin at most two. We will build, for each formula $C$, a diagram $D_{C}$ whose colimit will contain $R \stackrel{p_{c}}{\rightarrow} R$, where $p_{C}$ is the output polynomial of $C$. This will be done inductively on the size of $C$.

Each $D_{C}$ will be a diagram of the form

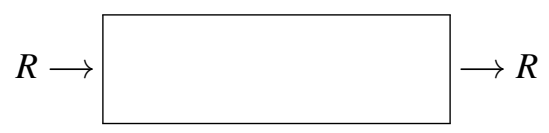

whose colimit is $R$ with the morphism from the $R$ on the right to the colimit being $\mathrm{id}_{R}$ and the morphism from the $R$ on the left to the colimit being defined by $1 \mapsto p_{C}$.

If the output $p_{C}$ of $C$ is one of the variables $x_{i}$, let $D_{C}$ be the diagram $R \stackrel{x_{i}}{\rightarrow} R$. If it is just a constant, then $D_{C}$ is $R \stackrel{c}{\rightarrow} R$.

If the top gate of $C$ is a product gate with $C^{\prime}$ and $C^{\prime \prime}$ as the left and right subcircuits, then we set $D_{C}$ by chaining together $D_{C^{\prime}}$ and $D_{C^{\prime \prime}}$ :

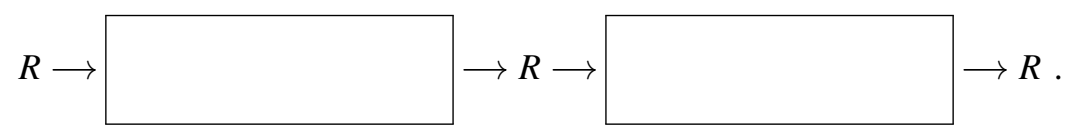

The map from the leftmost $R$ to the colimit is the composition $R \stackrel{p_{C^{\prime}}}{\longrightarrow} R \stackrel{p_{C^{\prime \prime}}}{\longrightarrow} R$, which is $R \stackrel{p_{C^{\prime}} p_{C^{\prime \prime}}}{\longrightarrow} R$.

If the top gate of $C$ is a sum gate with $C^{\prime}$ and $C^{\prime \prime}$ as the left and right subcircuits, then we define $D_{C}$ as 


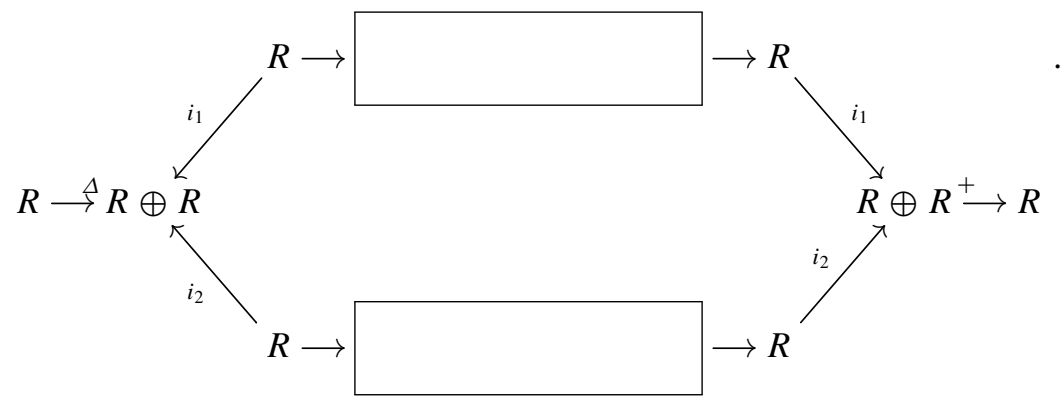

where the top and bottom rows are $D_{C^{\prime}}$ and $D_{C^{\prime \prime}}$. The colimit of this diagram is again $R$ with the map from the leftmost $R$ to the colimit being $p_{C}+p_{C^{\prime}}$.

What about a converse? What does the existence of a colimit computation in $R$-Mod that produces $R \stackrel{1 \mapsto f}{\longrightarrow} R$ say about the complexity of $f$ ?

THEOREM 5.7. Let $R=k\left[x_{1}, \ldots, x_{n}\right]$. If $R \stackrel{f}{\rightarrow} R$ is computed in a colimit computation with cost $c$ in $R$-Mod, then there is an arithmetic circuit of size poly $(c)$ with inputs $x_{1}, \ldots, x_{n}$, which computes $f$.

Proof. First of all note that $R$ is a unique factorization domain. Hence, given any finite tuple $\left(f_{1}, \ldots, f_{q}\right)$ of elements of $R$, there exists a unique (up to multiplication by units) greatest common divisor (GCD) of $f_{1}, \ldots, f_{q}$. We will need an algorithmic fact related to the computation of GCD in the proof below (cf. (5.3)).

Consider a diagram $D: I \rightarrow R$-Mod consisting only of the basic morphisms described above in (10). Assume that we have colim $D=R$.

For each vertex $v \in I$, we have that $D(v)$ is $R, R \oplus R$ or $\{0\}$. For each $v$ such that $D(v)=R$, let $f_{v}$ be the image of 1 under the morphism $R \stackrel{1 \mapsto f_{v}}{\longrightarrow} R$ from $D(v)$ to the colimit $R$. If $D(v)=\{0\}$, then we set $f_{v}=0$. If $D(v)=R \oplus R$, then we set two polynomials $f_{v}$ and $f_{v^{\prime}}$ so that the map $R \oplus R \rightarrow R$ to the colimit is given by $(1,0) \mapsto f_{v}$ and $(0,1) \mapsto f_{v^{\prime}}$. We will prove that each $f_{v}$ is computed by a polynomially sized circuit.

We are considering the $f_{v}$ 's as unknowns in a system of equations. For each arrow in $D$, we consider one or two $R$-linear equations. For an arrow $D\left(v_{1}\right) \rightarrow$ $D\left(v_{2}\right)$ of the form given in the left column, we add the equations in the right column:

$$
\begin{array}{ll}
R \stackrel{x_{i}}{\rightarrow} R & f_{v_{1}}-x_{i} f_{v_{2}} \\
R \stackrel{c}{\rightarrow} R & f_{v_{1}}-c f_{v_{2}}
\end{array}
$$




$$
\begin{aligned}
& R \stackrel{i_{1}, i_{2}}{\longrightarrow} R \oplus R f_{v_{1}}-f_{v_{2}} \text { or } f_{v_{1}}-f_{v_{2}^{\prime}} \\
& R \stackrel{\Delta}{\rightarrow} R \oplus R f_{v_{1}}-f_{v_{2}}-f_{v_{2}^{\prime}} \\
& R \oplus R \stackrel{+}{\rightarrow} R \quad f_{v_{1}}-f_{v_{2}} \text { and } f_{v_{1}^{\prime}}-f_{v_{2}} \\
& R \rightarrow\{0\} \quad f_{v_{1}}=0 \text { and } f_{v_{2}}=0 .
\end{aligned}
$$

In this way, we obtain a homogeneous system of $k\left[x_{1}, \ldots, x_{n}\right]$-linear equations; $A \vec{f}=0, A \in \operatorname{Mat}_{n_{2} \times s}(R)$. Tuples $\vec{f}=\left(f_{v_{1}}, f_{v_{2}}, f_{v_{3}}, \ldots, f_{v_{s}}\right) \in k\left[x_{1}, \ldots, x_{n}\right]^{s}$ that satisfy this system of equations correspond to cocones of the diagram $D$ with target $R$.

Since the colimit of $D$ is $R$, for any such cocone corresponding to $\left(f_{v_{1}}, \ldots, f_{v_{s}}\right)$, there will be a map (colim $D=R) \stackrel{1 \mapsto g}{\longrightarrow} R$ making the diagram containing the new cocone, the colimit cocone and the map $R \stackrel{1 \mapsto g}{\longrightarrow} R$ commute. This implies that $g$ divides each $f_{v_{j}}$. Since the colimit is the initial cocone, we can find the tuple of polynomials corresponding to the colimit cocone by taking $\left(\frac{f_{v_{1}}}{h}, \frac{f_{v_{2}}}{h}, \ldots\right.$, $\left.\frac{f_{v_{s}}}{h}\right)$, where $h=\operatorname{gcd}\left(f_{v_{1}}, \ldots, f_{v_{s}}\right)$. Thus, assuming the colimit is $R$, to compute the map from every $D(v)$ to the colimit, it suffices to: (i) find a solution to the above system of equations for $D$ and (ii) divide by $\operatorname{gcd}\left(f_{v_{1}}, \ldots, f_{v_{s}}\right)$.

To solve the system $A \vec{f}=0$, we use Gaussian elimination over the field $k\left(x_{1}, \ldots, x_{n}\right)$. Let $R$ and $C$ be square matrices with entries in $k\left(x_{1}, \ldots, x_{n}\right)$ such that $R A C$ is a diagonal matrix, in the sense that it contains an $r \times r$ minor that is $I_{r}$, with $r<s$, and all other entries are 0. Following the steps of Gaussian elimination, there exist circuits (or straight-line programs) with division that produce each entry of $R$ and $C$ in time poly $(s)$. Also, observe that the entries of $R$ and $C$ have degree at most $s$. Without loss of generality, assume $R A C e_{1}=0$. Then

$$
C e_{1}=\left(\frac{p_{1}}{q_{1}}, \ldots, \frac{p_{s}}{q_{s}}\right)^{T} \in k\left(x_{1}, \ldots, x_{n}\right)^{s}
$$

is a solution to $A \vec{f}=0$.

Before we proceed further and get this solution into $k\left[x_{1}, \ldots, x_{n}\right]^{s}$, we make a small digression into algorithms about straight-line programs. We consider the following, which are both proven by the algorithms in Kaltofen's work [Kal88]:

(GCD) Greatest common divisor: Given polynomials $f_{1}, \ldots, f_{q} \in k\left[x_{1}, \ldots, x_{n}\right]$, which are the output of a circuit of size $s^{\prime}$, there is a circuit of size poly $\left(s^{\prime}\right)$ that produces the GCD of $f_{1}, \ldots, f_{q}$. 
(DE) Denominator extraction: Given a reduced rational function

$$
\frac{p(x)}{q(x)} \in k\left(x_{1}, \ldots, x_{n}\right)
$$

produced by a circuit with division of size $s^{\prime}$, there is a circuit of size $\operatorname{poly}\left(s^{\prime}\right)$ that produces $q$.

In loc. cit., randomized algorithms that produce the output circuits for both DE and GCD are presented. But the circuits that output the gcd and the denominator are themselves not randomized. This will be enough in our nonuniform setting; we only use the existence of the polynomial size circuits that produce the gcd and the denominator and do not use the algorithm that produces the circuits themselves.

Going back to the proof, we made a system of equations $A \vec{f}=0$ from the diagram of basic morphisms and got a solution of the form

$$
C e_{1}=\left(p_{1} / q_{1}, \ldots, p_{s} / q_{s}\right)^{T} \in k\left(x_{1}, \ldots, x_{n}\right)^{s}
$$

and wanted to produce a solution in $k\left[x_{1}, \ldots, x_{n}\right]^{s}$ instead. To do this, first use Kaltofen's GCD to assume without loss of generality that each $\frac{p_{i}}{q_{i}}$ is reduced. Then use Kaltofen's DE to extract the denominators $q_{i}$ from each fraction. The element $q_{1} q_{2} \ldots q_{s} C e_{1}$ is in $k\left[x_{1}, \ldots, x_{n}\right]^{s}$ and is a solution to $A \vec{f}=0$. Now divide $q_{1} q_{2} \ldots q_{s} C e_{1}$ by the gcd of all of its entries to obtain $\left(f_{v_{1}}, \ldots, f_{v_{s}}\right) \in k\left[x_{1}\right.$, $\left.\ldots, x_{n}\right]^{s}$. The polynomials $f_{1}, \ldots, f_{s}$ correspond to the morphisms from $D$ to the colimit $R$. Each $f_{v}$ is produced by a circuit with division, but since the degrees of $f_{v}$ are polynomially bounded, each such circuit can be turned into a circuit without division using Strassen's method [Str73]. This concludes the proof that for any diagram of basic morphisms with $R$ as a colimit, every colimit cocone morphism from an object in $D$ sends 1 to an $f_{v}$, which is computed by a circuit of size polynomial in $s$.

We now prove the theorem. Let $R \stackrel{1 \mapsto f}{\longrightarrow} R$ be a subdiagram of a colimit computation with initial step $D_{0}$. Call the source $R_{1}$ and the target $R_{2}$. By Lemma 3.19, there is a subdiagram $D_{0}^{\prime} \subset D_{0}$ of basic morphisms whose colimit is $R_{1}$; and (cf. Remark 3.23) there is a subdiagram $D_{0}^{\prime \prime} \subset D_{0}$, which contains $D_{0}^{\prime}$, and whose colimit is $R_{2}$, with the induced map $R_{1} \rightarrow R_{2}$ being a map that sends $1 \mapsto f$. This implies, combined with the first part of this proof applied to both $D_{0}^{\prime}$ and $D_{0}^{\prime \prime}$, that $f$ is the quotient of two polynomials computed by polynomially sized circuits. Hence, by Strassen's method, $f$ is computed by a circuit of size polynomial in the size of $D_{0}$. 
REMARK 5.8. Note that the set of sequences of polynomials having formula sizes bounded by some quasipolynomial function (that is, a function of the form $n^{\log ^{c} n}$ ) coincides with the sequence of polynomials having arithmetic circuit complexity bounded by some quasipolynomial function over any field; that is, using the notation in [Bür00], $\mathrm{VQP}_{e}=\mathrm{VQP}$ [Bür00, Corollary 2.27]. Thus, Proposition 5.6 and Theorem 5.7 together imply that the colimit complexities of a sequence of polynomial morphisms $\left(k\left[x_{1}, \ldots, x_{n}\right] \stackrel{1 \mapsto f_{n}}{\longrightarrow} k\left[x_{1}, \ldots, x_{n}\right]\right)_{n>0}$ are bounded by a quasipolynomial function of $n$ if and only if the arithmetic circuit complexity of the sequence $\left(f_{n}\right)_{n>0}$ is also bounded by a quasipolynomial function.

\section{Functors}

In this section, we discuss the interplay of categorical complexity with functors between categories.

6.1. Preservation under functors. Let $F: \mathcal{C} \rightarrow \mathcal{D}$ be a functor. If $D$ is a diagram in $\mathcal{C}$, the image diagram $F(D)$ is defined in the obvious way. If $F$ preserves finite limits, then for every limit computation $\left(D_{0}, \ldots, D_{s}\right)$ in $\mathcal{C}$, starting with a set of basic morphisms $\mathcal{A}$ will correspond to a limit computation $\left(F\left(D_{0}\right), \ldots, F\left(D_{s}\right)\right)$ in $\mathcal{D}$, which starts with basic morphisms in $F(\mathcal{A})$. Therefore, in this case, we have

$$
c_{\mathcal{D}, F(\mathcal{A})}^{\lim }(F(D)) \leqslant c_{\mathcal{C}, \mathcal{A}}^{\lim }(D) .
$$

We have the analogous statement for colimit computations if $F$ preserves colimits.

Since equivalences preserve limits and colimits, categorical complexity, limit complexity, colimit complexity and mixed complexity are all invariant under equivalences of categories. A more general case is that of adjoint functors. Let $R: \mathcal{C} \rightarrow \mathcal{D}$ be a functor right-adjoint to a functor $L: \mathcal{D} \rightarrow \mathcal{C}$. Then, since right adjoints preserve limits and left adjoints preserve colimits, we have the following.

Lemma 6.1. Let $R: \mathcal{C} \rightarrow \mathcal{D}$ and $L: \mathcal{D} \rightarrow \mathcal{C}$ be a pair of adjoint functors. Let $\mathcal{A}$ be a set of basic morphisms in $\mathcal{C}$ and $\mathcal{A}^{\prime}$ be a set of basic morphisms in $\mathcal{D}$ such that $R(\mathcal{A}) \subset \mathcal{A}^{\prime}$ and $L\left(\mathcal{A}^{\prime}\right) \subset \mathcal{A}$. Then we have inequalities of complexities

$$
\begin{gathered}
c_{\mathcal{D}, \mathcal{A}^{\prime}}^{\lim _{1}}(R(D)) \leqslant c_{\mathcal{C}, \mathcal{A}}^{\lim _{1}}(D), \\
c_{\mathcal{C}, \mathcal{A}}^{\text {colim }}\left(L\left(D^{\prime}\right)\right) \leqslant c_{\mathcal{D}, \mathcal{A}^{\prime}}^{\text {colim }}\left(D^{\prime}\right),
\end{gathered}
$$

for every diagram $D$ in $\mathcal{C}$ and $D^{\prime}$ in $\mathcal{D}$. 
EXAMPLE 6.2. Let Vect $_{k}$ be the category of vector spaces over a field $k$. As the free vector space functor Fr : Set $\rightarrow$ Vect $_{k}$ is left-adjoint to the forgetful functor Fo : Vect $_{k} \rightarrow$ Set, we have that Fr preserves colimits. In particular, the colimit complexity of a vector space is bounded above by its dimension.

6.2. Complexity of functors. We discussed in Section 6.1 above, how certain functors preserve limits or colimits, and does also corresponding notions of complexities as well. In this section, we will study complexities of general functors. The first step is to define the complexity of a functor between categories.

Definition 6.3. A complexity function on a category $\mathcal{C}$ is a function that takes (finite) diagrams of $\mathcal{C}$ to $\mathbb{N} \cup\{\infty\}$.

EXAMPLE 6.4. For example, if $\mathcal{A}$ is a set of morphisms of $\mathcal{C}$ (assumed to have unit cost), then $c_{\mathcal{C}, \mathcal{A}}^{\lim }, c_{\mathcal{C}, \mathcal{A}}^{\text {colim }}, c_{\mathcal{C}, \mathcal{A}}^{\text {mixed }}$ are all examples of complexity functions on $\mathcal{C}$.

Definition 6.5 (Complexity of functors). Let $\mathcal{C}, \mathcal{D}$ be two categories with complexity functions, $\phi, \psi$, and let $F: \mathcal{C} \rightarrow \mathcal{D}$ be a functor. We define the complexity, $C_{\phi, \psi}(F): \mathbb{N} \rightarrow \mathbb{N}$ by

$$
C_{\phi, \psi}(F)(n)=\sup \left\{\psi(F(D)) \mid I \text { a finite directed graph, } D \in \mathcal{C}^{I}, \phi(D) \leqslant n\right\} \text {. }
$$

If $F$ is an endofunctor (that is, $\mathcal{C}=\mathcal{D}$ ) and $\phi=\psi$, then we will denote $C_{\phi, \psi}(F)$ just by $C_{\phi}(F)$.

One key example of functor complexity comes from image functors. In order to define the image functor, we first need to recall a few relevant notions from category theory.

6.3. Monomorphisms, slice categories, and the image functor. Let $\mathcal{C}$ be any category. Recall the following standard definitions.

Definition 6.6 (Monomorphisms). A morphism $f: A \rightarrow B$ in a category $\mathcal{C}$ is called a monomorphism if for all pairs of morphisms $g, h: C \rightarrow A, f \circ g=$ $f \circ h \Longrightarrow g=h$.

Definition 6.7 (Slice category). Let $A$ be an object of $\mathcal{C}$. The slice category, $\mathcal{C} / A$, is the category whose objects are morphisms $B \rightarrow A$, and whose morphisms 
are commutative diagrams:

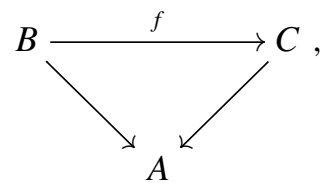

which compose in the obvious way.

The full subcategory of $\mathcal{C} / A$ consisting of monomorphisms $f: C \longmapsto A$ is denoted by $\operatorname{Sub}(A)$ (subobjects of $A$ ).

Definition 6.8. We say that a category $\mathcal{C}$ has images if for every object $A$ of $\mathcal{C}$, the inclusion functor $\mathrm{i}_{\mathcal{C}, A}: \operatorname{Sub}(A) \rightarrow \mathcal{C} / A$ has a left adjoint $\operatorname{im}_{\mathcal{C}, A}$ (cf. [Joh02, Lemma 1.3.1]).

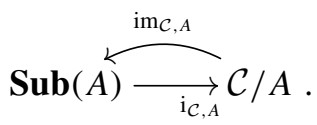

REMARK 6.9. Note that the adjointness $\operatorname{im}_{\mathcal{C}, A} \dashv \mathrm{i}_{\mathcal{C}, A}$ is equivalent to saying that given a monomorphism $f: B \longmapsto A$ and a morphism $g: C \rightarrow A$ in $\mathcal{C} / A$, there is a natural isomorphism

$$
\mathcal{C} / A\left(g, \mathrm{i}_{\mathcal{C}, A}(f)\right) \cong \mathbf{S u b}(A)\left(\operatorname{im}_{\mathcal{C}, A}(g), f\right),
$$

where the isomorphism takes $\theta \in \mathcal{C} / A\left(g, \mathrm{i}_{\mathcal{C}, A}(f)\right)$ given by the diagram

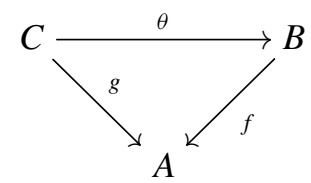

to the element in $\operatorname{Sub}(A)\left(\operatorname{im}_{\mathcal{C}}(g), f\right)$ described by the diagram

$$
\operatorname{dom}\left(\operatorname{im}_{\mathcal{C} / A}(g)\right) \stackrel{\operatorname{im}_{\mathcal{C} / A}(\theta)}{\longrightarrow} B .
$$

In particular, taking $f=\operatorname{im}_{\mathcal{C}, A}(g)$, the image under the inverse of the isomorphism defined above of the element $1_{f} \in \mathbf{S u b}(A)$ induces a morphism $\varepsilon(g): B \rightarrow \operatorname{dom}\left(\operatorname{im}_{\mathcal{C} / A}(f)\right)$, making the following diagram commute: 


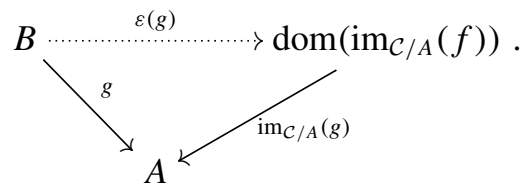

We now extend the image functor from the slice categories $\mathcal{C} / A$ to the diagram category $\mathcal{C}^{\bullet \rightarrow \bullet}$.

Proposition 6.10. Suppose that $\mathcal{C}$ is a category that has pullbacks and images. Then in the diagram category $\mathcal{C}^{\bullet \rightarrow \bullet}$, letting $\operatorname{Mon}_{\mathcal{C}}$ denote the full subcategory of monomorphisms, and $\mathrm{i}_{\mathcal{C}}: \operatorname{Mon}_{\mathcal{C}} \rightarrow \mathcal{C}^{\bullet} \rightarrow \bullet$ the inclusion functor, there exists a left adjoint $\mathrm{im}_{\mathcal{C}}$ to $\mathrm{i}_{\mathcal{C}}$.

Proof. The functor $\operatorname{im}_{\mathcal{C}}$ is defined as follows. For an object $f: C \rightarrow A$ of $\mathcal{C} \bullet \bullet \bullet$, we set $\operatorname{im}_{\mathcal{C}}(f)=\operatorname{im}_{\mathcal{C} / A}(f)$. For a morphism, $\theta=\left(\theta_{0}, \theta_{1}\right)$ of $\mathcal{C} \bullet \bullet \bullet$, given by the following commutative diagram,

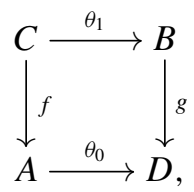

consider the pullback diagram

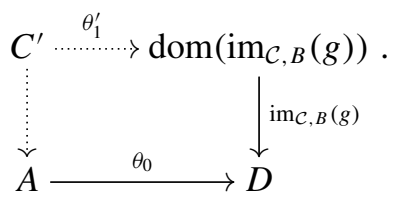

Since, the morphism $\operatorname{im}_{\mathcal{C}, B}(g) \operatorname{dom}\left(\operatorname{im}_{\mathcal{C}, B}(g)\right) \rightarrow D$ is a monomorphism, and the pullback of a monomorphism is again a monomorphism, the left vertical arrow is a monomorphism. Moreover, from the universal property of the pullback applied to the diagram

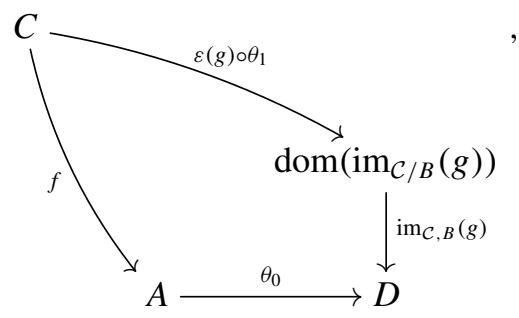


we obtain the commuting diagram

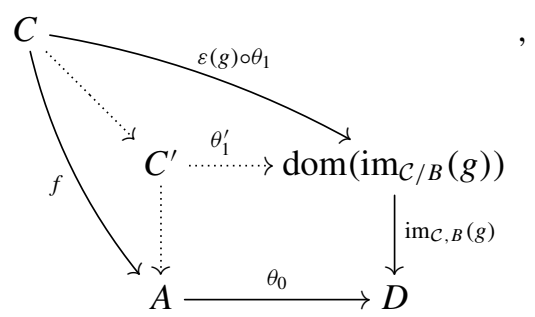

where the dotted diagonal arrow is isomorphic to $\varepsilon(f): C \rightarrow \operatorname{dom}\left(\operatorname{im}_{\mathcal{C} / A}(f)\right)$ (using the fact that the left vertical arrow is a monomorphism). We set $\operatorname{im}_{\mathcal{C}}(\theta)=$ $\theta^{\prime}=\left(\theta_{1}^{\prime}, \theta_{0}\right) \in \operatorname{Mon}_{\mathcal{C}}\left(\operatorname{im}_{\mathcal{C}}(f), \operatorname{im}_{\mathcal{C}}(g)\right)$.

To check $\operatorname{im}_{\mathcal{C}}$ is left-adjoint to $i_{\mathcal{C}}$ is an easy (if tedious) exercise.

EXAMPLE 6.11 (Examples of categories with images). Most of the categories introduced before have images (and also pullbacks). It is easy to verify that the categories Vect $_{k}$, Set, Top, SL, SA have images as well as pullbacks. The fact that the last two categories (see Notation 2.3, Part D for their definitions) have images is a consequence of the fact that the images of semilinear (respectively, semialgebraic) sets are closed under taking images under affine (respectively, polynomial) maps.

6.4. Complexity of the image functor. The $P$ versus NP question in computational complexity (say, in the real B-S-S model of computation) is fundamentally about comparing the complexities of sequences of semialgebraic sets, which belong to an 'easy' class (that is, the B-S-S complexity class $\mathrm{P}_{\mathbb{R}}$ ), with the complexities of sequences obtained by taking images under certain projections of sequences belonging to the 'easy' class (by taking the images under projections of sequences in the class $\mathrm{P}_{\mathbb{R}}$, one obtains the B-S-S complexity class $\mathrm{NP}_{\mathbb{R}}$ ). A formal definition of $\mathrm{P}_{\mathbb{R}}, \mathrm{NP}_{\mathbb{R}}$ (and indeed of the whole polynomial hierarchy $\left.\mathrm{PH}_{\mathbb{R}}\right)$ can be found in [BZ10, §1.2.1] and will not be repeated here. (We only note that existential quantifiers used in the definition of $\mathrm{NP}_{\mathbb{R}}$ in [BZ10] geometrically correspond to taking the image under projection, or more precisely, elimination of existential quantifiers geometrically corresponds to taking the image under a linear projection map.) By replacing the notion of $\mathrm{B}-\mathrm{S}-\mathrm{S}$ complexity by other nonuniform notions of complexity, one can extend these definitions to the nonuniform case as well (see [Isi19] and [BP18]). Finally, what is perhaps closest to the point of view of this paper, in [Bas15], a sheaf theoretic analog of the classes $\mathrm{P}_{\mathbb{R}}$ and $\mathrm{NP}_{\mathbb{R}}$ is defined. The elements of these classes 


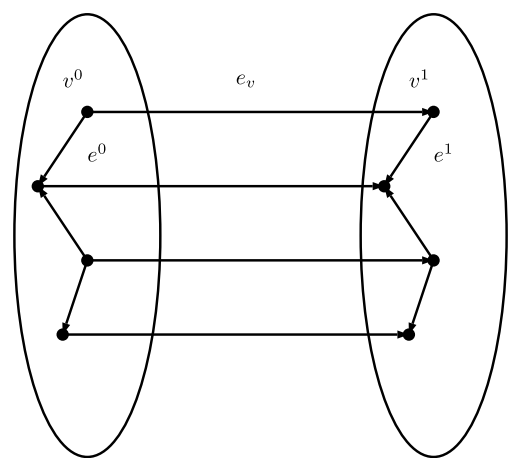

Figure 9. The directed graph $I^{\prime}$.

are no longer sequences of semialgebraic sets, but rather of semialgebraically constructible sheaves, and the role of taking images under projection maps is replaced by the direct image functor. A ' $\mathrm{P}$ versus NP' conjecture in this general setting is formulated in [Bas15] (Conjecture 3.83). It should be clear from the above discussion that the P versus NP question is intimately related to studying how badly the complexity of certain objects (say semialgebraic sets) blows up (that is, whether the blow-up is polynomially bounded or not) after taking the image under certain tame maps. The notion of 'complexity' of a sequence of sets in the formulations described above is different in each case, but is related for example to the number of steps taken by a B-S-S machine, or the size of an arithmetic circuit, required for testing membership in the corresponding sequence of sets.

With the discussion above as background, we proceed to define an analog of the $P$ versus NP question in the categorical setting. The main idea is to replace 'taking images under projections' by the image functor in the category under question (assuming it has an image functor) and replacing the notion of 'complexity' by categorical complexity. The categorical analog turns out to be simpler, coordinate independent, and also free of taking sequences.

In this paper, we do not attempt to prove any formal relationships between the categorical and standard versions of the $\mathrm{P}$ versus NP question, leaving such questions for future investigations. However, we hope the discussion in the previous paragraph makes clear the analogy between the standard ' $P$ versus NP' questions, with the categorical analog.

First, observe that for any category $\mathcal{C}$, a diagram $D: I=(V, E, s, t) \rightarrow \mathcal{C}^{\bullet} \rightarrow \bullet$ in the diagram category of $\mathcal{C}^{\bullet} \rightarrow \bullet$ induces a diagram $D^{\prime}: I^{\prime}=\left(V^{\prime}, E^{\prime}, s^{\prime}, t^{\prime}\right) \rightarrow \mathcal{C}$ as follows (cf. Figure 9). 
Here $V^{\prime}=V^{0} \cup V^{1}$, where $V^{i}=\left\{v^{i} \mid v \in V\right\}$ is a copy of $V$ for $i=0,1$, and $E^{\prime}=E^{0} \cup E^{1} \cup \bigcup_{v \in V}\left\{e_{v}\right\}$, where $E^{i}=\left\{e^{i} \mid e \in E\right\}$ is a copy of $E$ for $i=0,1$, and

$$
\begin{aligned}
& \left.\begin{array}{l}
s^{\prime}\left(e^{i}\right)=s(e)^{i} \\
t^{\prime}\left(e^{i}\right)=t(e)^{i}
\end{array}\right\} \quad \text { for } e \in E, i=0,1, \\
& \left.\begin{array}{l}
s^{\prime}\left(e_{v}\right)=v^{0}, \\
t^{\prime}\left(e_{v}\right)=v^{1}
\end{array}\right\} \quad \text { for } v \in V,
\end{aligned}
$$

and

$$
\begin{aligned}
& D^{\prime}\left(e^{0}\right)=D(s(e)) \\
& D^{\prime}\left(e^{1}\right)=D(t(e)) \\
& D^{\prime}\left(e_{v}\right)=D(v), v \in V
\end{aligned}
$$

remembering that for each vertex $v \in V, D(v)$ is a morphism of $\mathcal{C}$.

Using the notation introduced above, we have the following definition.

Definition 6.12 (Image complexity). If $\mathcal{A}$ is a set of morphisms in $\mathcal{C}$, we will denote for $*=\lim$, colim, mixed for any diagram $D$ of $\mathcal{C}^{\bullet \rightarrow \bullet}$ (respectively, $\operatorname{Mon}_{\mathcal{C}}$ ); we define the image complexity $c_{\mathcal{C} \bullet \bullet \cdot, \mathcal{A}}^{*}(D)$ (respectively, $\left.c_{\text {Mon }_{\mathcal{C}}, \mathcal{A}}^{*}(D)\right)$ of the diagram $D$ by

$$
c_{\mathcal{C} \bullet \rightarrow \cdot \mathcal{A}}^{*}(D)=c_{\mathcal{C}, \mathcal{A}}^{*}\left(D^{\prime}\right)
$$

(respectively, $c_{\operatorname{Mon}_{\mathcal{C}, \mathcal{A}}^{*}}(D)=c_{\mathcal{C}, \mathcal{A}}^{*}\left(D^{\prime}\right)$ ), where the right-hand side is the (limit, colimit or mixed) complexity of the diagram $D^{\prime}$ in the category $\mathcal{C}$ (cf. Definition 3.13), with $\mathcal{A}$ as the set of basic morphisms.

Now suppose that a category $\mathcal{C}$ has images and $\mathcal{A}$ is a set of morphisms of $\mathcal{C}$. Recall the definition of the complexity of a functor between two categories with complexity functions (cf. Definition 6.5). Applying Definition 6.5 to the functor

$$
\operatorname{im}_{\mathcal{C}}: \mathcal{C}^{\cdot \rightarrow \cdot} \rightarrow \operatorname{Mon}_{\mathbb{C}}
$$

with complexity functions $c_{\mathcal{C}^{\bullet} \rightarrow \cdot, \mathcal{A}}^{*}$ and $c_{\mathrm{Mon}_{\mathcal{C}, \mathcal{A}}}^{*}$ (with $*=\lim$, colim, mixed), on the source and target of the functor $\operatorname{im}_{\mathcal{C}}$, we obtain the function

$$
C_{c_{\mathcal{C} \bullet \rightarrow \cdot, \mathcal{A}}^{*}, c_{\mathrm{Mon} \mathcal{C}, \mathcal{A}}^{*}}\left(\operatorname{im}_{\mathcal{C}}\right)(n) .
$$

DEFINITION 6.13 (Complexity of the image functor). With $*=\mathrm{lim}$, colim, mixed, we will denote

$$
\operatorname{IFC}_{\mathcal{C}, \mathcal{A}}^{*}(n)=C_{c_{\mathcal{C} \bullet \bullet \cdot \mathcal{A}}^{*}, c_{\mathrm{Mon}_{\mathcal{C}}, \mathcal{A}}^{*}}\left(\operatorname{im}_{\mathcal{C}}\right)(n)
$$


and call $\mathrm{IFC}_{\mathcal{C}, \mathcal{A}}^{*}$ the $*$-complexity of the image functor of the category $\mathcal{C}$ (with respect to the basic set of morphisms $\mathcal{A}$ ).

Now for any category $\mathcal{C}$ that has images, and for any subset $\mathcal{A}$ of morphisms of $\mathcal{C}$, and $*=\lim$, colim, mixed, we can ask the following question.

QUESTION 6.14. Is the function

$$
\operatorname{IFC}_{\mathcal{C}, \mathcal{A}}^{*}
$$

bounded by some polynomial?

6.5. The image functor in the categories SL and SA. First, observe that the class of semilinear (respectively, semialgebraic) sets is closed under taking images of affine (respectively, polynomial) maps. This is a consequence of FourierMotzkin elimination (respectively, Tarski-Seidenberg principle). However, the answer to Question 6.14 may depend on the type of categorical complexity that is being considered.

We consider the cases of limit and mixed complexity, starting with the limit complexity in the categories SL and SA. We prove the answer to Question 6.14 in SL and SA for limit complexity with the choice of the basic morphisms defined below. This should not be too much of a surprise since the power of limit computation with these choices of basic morphisms is quite limited. Only closed convex polyhedral subsets of $\mathbb{R}^{n}$ can be constructed in SL (respectively, basic closed semialgebraic sets) using limit computations. (A basic closed semialgebraic set is a semialgebraic set defined by a conjunction of a finite number of weak polynomial inequalities of the form $P \geqslant 0$.) Moreover, in the case of the category $\mathbf{S L}$, we prove a polynomial upper bound on the number of facets of an object in terms of limit complexity (Lemma 6.15 below). The negative answer to Question 6.14 in this case can be deduced by exhibiting a sequence of morphisms of polynomially bounded complexity such that the number of facets of the image grows super-polynomially. We exhibit such a sequence using the wellknown properties of cyclic polytopes. In the case of the category SA, the situation is even simpler since it is well known that images under polynomial maps (in fact, projections along a coordinate) of a basic closed semialgebraic set need not be basic closed. We exhibit a simple example of this phenomenon.

6.5.1. Limit complexity of the image functor in SL. Recall that the objects of the category SL are (embedded) semilinear sets and morphisms between such sets, which are restrictions of affine mappings. Let $\mathcal{A}$ consist of the scalar 
multiplication morphisms $\mathbb{R} \stackrel{c}{\rightarrow} \mathbb{R}$ for each $c \in \mathbb{R}$, the addition morphism $\mathbb{R}^{2} \stackrel{+}{\rightarrow} \mathbb{R}$, and morphisms $[0, \infty) \hookrightarrow \mathbb{R}, \mathbb{R} \rightarrow 0$. It is easy to see that using limit computations, one can produce every morphism $f:\left(A \subset \mathbb{R}^{m}\right) \rightarrow\left(B \subset \mathbb{R}^{n}\right)$, where $A, B$ are closed polyhedral subsets of $\mathbb{R}^{m}$ and $\mathbb{R}^{n}$, respectively.

LEMMA 6.15. The number of facets of each object $A$ is bounded from above by $\left(c_{\mathbf{S L}, \mathcal{A}}^{\lim }(A)\right)^{2}$.

Proof. Let $\left(D_{0}, \ldots, D_{s}\right)$ be the sequence of diagrams of a limit computation of $A$. We prove by an induction on $s$ the following statement from which the lemma will follow.

For each vertex $v$ of $D_{s}$, let $h_{s}(v)=\operatorname{card}\left(H_{s}(v)\right)$, where $H_{s}(v)$ is the set of vertices of $D_{s}$ which was introduced by taking a limit, and which has $v$ in its cone. We follow the convention that if $v$ is a vertex of $D_{0}$, then $H_{s}(v)=\{v\}$. Because of the constructivity assumption, $h_{s}(v)$ is well defined.

We claim that the number of facets of $A$ is bounded by $\sum_{v \in D_{s}} h_{s}(v)$. We prove the claim by an induction on $s$. The claim is clearly true if $s=0$ since the objects that are domains or codomains of the basic morphisms have at most one facet, and $h_{s}(v)=1$ for each vertex of $D_{0}$. Now, assume that the claim holds for all $s^{\prime}<s$.

For each vertex $v$ of $D_{s-1}$, let $A_{v}=D_{s-1}(v)$. Let $V_{s-1}$ be the set of vertices of $D_{s-1}$, which do not belong to the limit cone of any vertex $v$ of $D_{s-1}$ not equal to itself. Then, $A$ is the intersection of the product of the polytopes $A_{v}, v \in V_{s-1}$ with an affine subspace. This implies that the number of facets of $A$ is bounded by the sum of the number of facets of $A_{v}, v \in V_{s-1}$. Now use the induction hypothesis to finish the proof of the claim.

It is clear that the lemma follows from the claim since for each vertex $v$ of $D_{s}$, $h_{s}(v)$ is bounded by the total number of vertices of $D_{s}$, which itself is bounded by the cost of the diagram computation $\left(D_{0}, \ldots, D_{s}\right)$.

Consider now the following example. Denote by $\theta_{m}(t)=\left(t, t^{2}, \ldots, t^{2 m}\right) \subset \mathbb{R}^{2 m}$ the moment curve in $\mathbb{R}^{2 m}$. The convex hull of $n$ distinct points on the moment curve is called a cyclic polytope, $\operatorname{Cyl}(n, m)$. For $n>2 m$, the number of facets of $\operatorname{Cyl}(n, m)$ is given by [Gal63, Theorem 4]

$$
\left(\begin{array}{c}
n-m \\
m
\end{array}\right)+\left(\begin{array}{c}
n-m-1 \\
m-1
\end{array}\right)
$$

In particular, the number of facets of $\mathrm{Cyl}(4 m+1,2 m)$ equals $4 m$. It is easy to derive that the limit complexity of $\operatorname{Cyl}(4 m+1,2 m)$ is bounded by $O\left(m^{2}\right)$. Now let $\pi_{m}: \mathbb{R}^{4 m} \rightarrow \mathbb{R}^{2 m}$ be the projection on the first $2 m$ coordinates. Clearly, $\pi_{m}(\operatorname{Cyl}(4 m+1,2 m))=\operatorname{Cyl}(4 m+1, m)$, and the number of facets 
of Cyl $(4 m+1, m)$ equals

$$
\left(\begin{array}{c}
3 m+1 \\
m
\end{array}\right)+\left(\begin{array}{c}
4 m \\
m-1
\end{array}\right)
$$

which is clearly exponential in $m$. It follows from Lemma 6.15 that the limit complexity of $\pi_{m}(\mathrm{Cyl}(4 m+1,2 m))$ is exponentially large in $m$.

Thus, the object $\pi_{m}: \operatorname{Cyl}(4 m+1,2 m) \rightarrow \mathbb{R}^{2 m}$ of the category $\mathbf{S L}^{\bullet \rightarrow \bullet}$ has limit complexity polynomially bounded in $m$; the limit complexity of $\left(i m_{\mathbf{S L}}\right)\left(\pi_{m}\right)$ is exponentially large in $m$. It follows from this example the next proposition.

Proposition 6.16. The function $\mathrm{IFC}_{\mathrm{SL}, \mathcal{A}}^{\lim }$ is not polynomially bounded (where $\mathcal{A}$ consists of the scalar multiplication morphisms $\mathbb{R} \stackrel{c}{\rightarrow} \mathbb{R}$ for each $c \in \mathbb{R}$, the addition morphism $\mathbb{R}^{2} \stackrel{+}{\rightarrow} \mathbb{R}$, and morphisms $[0, \infty) \hookrightarrow \mathbb{R}, \mathbb{R} \rightarrow 0$ ).

6.5.2. Limit complexity of the image functor in SA. The case of the category SA with respect to limit complexity is simpler. Recall that the objects of the category SA are (embedded) semialgebraic sets and morphisms between such sets, which are restrictions of polynomial mappings. Let $\mathcal{A}$ consist of the scalar multiplication morphisms $\mathbb{R} \stackrel{c}{\rightarrow} \mathbb{R}$ for each $c \in \mathbb{R}$, the addition morphism $\mathbb{R}^{2} \stackrel{+}{\rightarrow} \mathbb{R}$, the multiplication morphism $\mathbb{R}^{2} \rightarrow \mathbb{R}$, and morphisms $[0, \infty) \hookrightarrow \mathbb{R}, \mathbb{R} \rightarrow 0$. It is not difficult to see that the objects of SA that can be constructed using a limit computation are exactly the basic closed semialgebraic sets. On the other hand, it is well known that the image under polynomial maps (for example, projections along some coordinates) of a basic closed semialgebraic set need not be a basic closed semialgebraic set. For example, consider the real variety $V$ defined by

$$
\left(X_{1}-X_{3}^{2}\right)\left(X_{2}-X_{4}^{2}\right)=0 \text {. }
$$

Denoting by $\pi: \mathbb{R}^{4} \rightarrow \mathbb{R}^{2}$ the projection to $X_{1}, X_{2}$-coordinates, $\pi(V)=\left\{\left(x_{1}, x_{2}\right)\right.$ $\left.\in \mathbb{R}^{2} \mid x_{1} \geqslant 0 \vee x_{2} \geqslant 0\right\}$, which is not a basic closed semialgebraic set (as observed by Lojasiewicz; see [AR94, page 466]), and hence $\pi(V)$ has infinite limit complexity.

The next proposition follows.

PROPOSITION 6.17. The function $\mathrm{IFC}_{\mathbf{S A}, \mathcal{A}}^{\lim }$ is not polynomially bounded (where $\mathcal{A}$ consists of the scalar multiplication morphisms $\mathbb{R} \stackrel{c}{\rightarrow} \mathbb{R}$ for each $c \in \mathbb{R}$, the addition morphism $\mathbb{R}^{2} \stackrel{+}{\rightarrow} \mathbb{R}$, the multiplication morphism $\mathbb{R}^{2} \rightarrow \mathbb{R}$, and morphisms $[0, \infty) \hookrightarrow \mathbb{R}, \mathbb{R} \rightarrow 0)$. 
6.5.3. Complexity of the image functor with respect to mixed complexity in SL and SA. Mixed computation is much more powerful than limit computation, and we are unable to resolve the following question, which we posit as the categorical analog of the P versus NP question in the categories SL and SA (refer to the discussion in the beginning of this section).

QUESTION 6.18. Are the functions $\operatorname{IFC}_{\mathrm{SL}, \mathcal{A}}^{\text {mixed }}, \operatorname{IFC}_{\mathrm{SA}, \mathcal{A}}^{\text {mixed }}$ polynomially bounded (with the set of morphisms $\mathcal{A}$ being the same as in Propositions 6.16 and 6.17, respectively)?

We also make the following conjecture.

CONJECTURE 6.19. The function $\mathrm{IFC}_{\mathbf{S A}, \mathcal{A}}^{\text {mixed }}$ is bounded singly exponentially.

This should be thought of as the categorical analog of the existence of algorithms with singly exponential algorithms for the elimination of one block of existential quantifiers in the first-order theory of the reals (see for example, [BPR06]).

\subsection{The image functor in the category $k\left[x_{1}, \ldots, x_{n}\right]$-Mod and colimit} complexity. We have considered complexity of the image functor with respect to limit and mixed complexity in several categories. We now consider the image functor from the point of view of colimit complexity in the category $R$-Mod, where $R=k\left[x_{1}, \ldots x_{n}\right]$, with the set basic morphisms defined in (10).

We describe a method for writing a colimit computation to compute the image of morphism of modules. A careful analysis of the part of the proof of Proposition 6.20, which makes use of the existence of Gröbner basis of modules, would give an upper bound for the complexity of the image functor in $R$-Mod. With a naive analysis, we can only obtain an unnecessarily high bound (doubly exponential), so we leave the complexity analysis (that is the explicit dependence on the parameter $s$ ) out of the following statement.

Proposition 6.20. Let $M \stackrel{\varphi}{\rightarrow} N$ be a morphism diagram in $R$-Mod, computed by a colimit computation of size $s$. Then, there is a colimit computation that computes $\operatorname{im}(\varphi) \rightarrow N$ of size bounded by some function of $s$.

Proof. By Lemma 3.19 and Remark 3.23, the colimit computation that produces $M \stackrel{\varphi}{\rightarrow} N$ can be simplified to produce (only) $M \stackrel{\varphi}{\rightarrow} N$ in two colimit steps. The first one is taking the colimit of diagram $D_{M}$ of basic morphisms, which produces $M$. The second one is the colimit of a larger diagram $D_{N} \supset D_{M}$ together with $M$ 
and all the cocone morphisms from the objects in $D_{M}$ to $M$. Replacing colimits with coequalizers and coproducts, we have that $M$ is isomorphic to the quotient

$$
\bigoplus_{\rho \in s\left(D_{M}\right)} R^{j_{\rho}} \stackrel{A_{M}}{\longrightarrow} \bigoplus_{\gamma \in \mathrm{v}\left(D_{M}\right)} R^{j_{\gamma}} \longrightarrow M
$$

where $\gamma$ runs over all the vertices in $D_{M}$ and $\rho$ runs over all sources of arrows in $D_{M}$; and $j_{\rho}$ and $j_{\gamma}$ are 0,1 , or 2 , based on whether the corresponding basic object is $\{0\}, R$, or $R^{2}$. (Note that when using Remark 3.23, we are implicitly using the fact that in the category $R$-Mod, the coequalizer of two morphisms $\phi, \psi: A \rightarrow B$ is isomorphic to the cokernel of $\phi-\psi$.)

Similarly we can write $N$ as a quotient,

$$
\bigoplus_{\rho \in s\left(D_{N}\right)} R^{j_{\rho}} \oplus \bigoplus_{v \in \mathrm{v}\left(D_{M}\right)} R^{j_{v}} \longrightarrow \bigoplus_{\gamma \in \mathrm{v}\left(D_{N}\right)} R^{j_{\gamma}} \oplus M \longrightarrow N
$$

Since $M$ is the colimit of $D_{M}$, we can remove the extra sum on the left and the $M$ summand in the middle. So $N$ is the quotient:

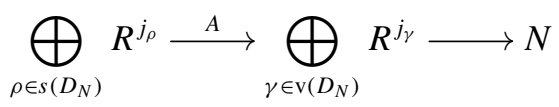

Combining the direct sums, we get a commuting diagram with exact rows:

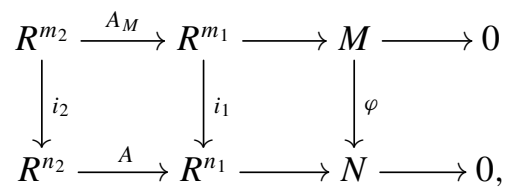

where the maps $i_{1}$ and $i_{2}$ are the inclusion of the first $m_{1}$ and $m_{2}$ coordinate spaces, respectively. Written this way, the map $\varphi$ from $M$ to $N$ is induced by the inclusion of the generators of $M$ into the generators of $N$.

To construct the image $\operatorname{im}(\varphi)$, we need to find the relations among the images of the generators of $M$, that is, the first $m_{1}$ generators of $N$. It is possible to use Gröbner basis methods to obtain a presentation of $\operatorname{im}(\varphi)$, that is, obtain a $B=m_{1} \times u$ matrix with entries in $R$ such that

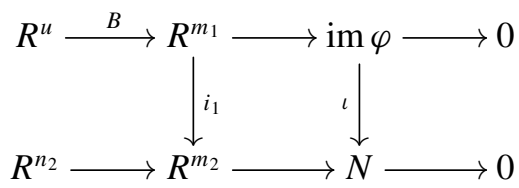


(see for instance [KR00, Proposition 3.3.1, Part (b)]). The size of the matrix $B$ and the degrees of the polynomials appearing in it are bounded by some explicit (possibly doubly exponential or worse) function of the degrees of the polynomials appearing in the matrix $A$ coming from the analysis of algorithms for computing Gröbner basis of submodules of a free $R$-module given a set of generators. The important point for us is that these degrees and the size of $B$ are thus bounded by some function of $s$ (the colimit complexity of the given morphism $M \stackrel{\varphi}{\rightarrow} N$ ).

It is easy to see that using the basic morphisms and colimits, one can realize using a colimit computation any $R$ mod homomorphisms between two free $R$ modules of finite rank, with cost depending on the degrees of the polynomials appearing in the matrix corresponding to the homomorphism. Thus, we can obtain the homomorphism $\mathbb{R}^{u} \stackrel{B}{\rightarrow} \mathbb{R}^{m_{1}}$ using colimit computation of size bounded by some function of $s$.

We can now obtain the $R$-module $\operatorname{im}(\varphi)$ using a colimit computation by taking the colimit of the following diagram:

$$
0 \leftarrow R^{u} \stackrel{B}{\rightarrow} R^{m_{1}} .
$$

The inclusion homomorphism $\iota: \operatorname{im}(\varphi) \stackrel{\iota}{\rightarrow} N$ can be computed by constructing $N$ again by taking the basic object $R$ 's in the computation of $\operatorname{im}(\varphi)$ corresponding to the generators of $M$ and constructing any remaining generators and relations for $N$ from (12).

\section{Open problems and future directions}

In this section, we list some open problems suggested by the contents of this paper.

(1) Resolve Questions 5.1 and 5.5.

(2) Prove singly exponential upper bound on the mixed complexity of the image functor in the semialgebraic category. This would correspond to the singly exponential complexity algorithms for computing the image of semialgebraic sets under polynomial maps, which follow from the critical point method in algorithmic real algebraic geometry (see for example, [BPR06, Ch. 14]).

(3) Prove singly exponential upper bound on the categorical complexity of the image functor in the category $R$-Mod (cf. Proposition 6.20).

(4) One important research direction in quantitative semialgebraic geometry has been to prove tight bounds on the topological complexity of 
semialgebraic sets in terms of various parameters bounding the sizes of the formulas defining them-such as the number of polynomial inequalities, their degrees or the number of monomials in their support, or even the additive complexity (see for example, [BPR05]). One could ask for similar results in the categorical setting. In the language introduced in this paper, this would mean investigating the complexity of the homology functor $\mathrm{H}: \mathbf{S A} \rightarrow \mathbb{Z}$-Mod.

(5) Investigate the relationship between the B-S-S complexity classes over the real numbers and the (mixed)-categorical complexity of objects in SA.

(6) Prove or disprove that the image functor in various categories has polynomially bounded (mixed) complexity. This is the categorical version of the $\mathrm{P}$ versus NP question in ordinary complexity theory (see Questions 6.14 and 6.18).

(7) Note that the image functor $\operatorname{im}_{\mathcal{C}}$ has a right adjoint $i_{\mathcal{C}}$ whose complexity is clearly bounded polynomially (in fact, $C_{\mathrm{i}_{\mathcal{C}}, \mathcal{A}}(n)=n$ for any set $\mathcal{A}$ of basic morphisms in $\mathcal{C}$ ). More generally, functors often come in adjoint pairs, and often it is easy to show that one of them has polynomially bounded complexity, while the other is conjecturally hard (that is, not polynomially bounded). More examples are given in the category of constructible sheaves in [Bas15]. As already noted, left-adjoint functors preserve colimits and thus are well behaved with respect to colimit computations, while rightadjoint ones preserve limits and are well behaved with respect to limit computations. It will be interesting to analyze the role of adjointness on mixed limit-colimit computations in different categories.

(8) Extend the notion of categorical and functor complexity to categories that are further enriched-for example, triangulated categories, derived categories, and so on. This would enable one to study for example the complexity of sheaves in the derived category and various natural functors (for example, the six operations of Grothendieck) from the point of view of categorical complexity. A first attempt toward studying the complexity of constructible sheaves and their functors is undertaken in [Bas15]. However, the definition of complexity in [Bas15] is not categorical.

\section{Acknowledgements}

The first author was supported in part by NSF grants CCF-1319080, CCF1618981, DMS-1620271, and CCF-1910441 while working on this paper. 


\section{Conflict of Interest: None.}

\section{References}

[AR94] C. Andradas and J. M. Ruiz, 'Ubiquity of Lojasiewicz's example of a nonbasic semialgebraic set', Michigan Math. J. 41(3) (1994), 465-472. MR 1297702.

[Awo10] S. Awodey, Category Theory (Oxford University Press, Oxford, UK, 2010).

[Bas15] S. Basu, 'A complexity theory of constructible functions and sheaves', Found. Comput. Math. 15(1) (2015), 199-279.

[BP18] S. Basu and D. Patel, 'Connectivity of joins, cohomological quantifier elimination, and an algebraic Toda's theorem', Preprint, 2018, arXiv:1812.07483.

[BPR05] S. Basu, R. Pollack and M.-F. Roy, 'Betti number bounds, applications and algorithms', in Current Trends in Combinatorial and Computational Geometry: Papers from the Special Program at MSRI (Cambridge University Press, Cambridge, UK, 2005), 87-97. MSRI Publications, 52.

[BPR06] S. Basu, R. Pollack and M.-F. Roy, Algorithms in Real Algebraic Geometry, Algorithms and Computation in Mathematics, 10 (Springer, Berlin, 2006), MR 1998147 (2004g:14064).

[BZ10] S. Basu and T. Zell, 'Polynomial hierarchy, Betti numbers, and a real analogue of Toda's theorem', Found. Comput. Math. 10(4) (2010), 429-454. MR 2657948.

[BGMW11] E. Bierstone, D. Grigoriev, P. Milman and J. Włodarczyk, 'Effective Hironaka resolution and its complexity', Asian J. Math. 15(2) (2011), 193-228. MR 2838220.

[BCS97] P. Bürgisser, M. Clausen and M. Amin Shokrollahi, Algebraic Complexity Theory, Grundlehren der Mathematischen Wissenschaften [Fundamental Principles of Mathematical Sciences], 315 (Springer, Berlin, 1997), With the collaboration of Thomas Lickteig. MR 1440179.

[BCSS98] L. Blum, F. Cucker, M. Shub and S. Smale, Complexity and Real Computation (Springer, New York, 1998), With a foreword by Richard M. Karp. MR 1479636 (99a:68070).

[Bür00] P. Bürgisser, Completeness and Reduction in Algebraic Complexity Theory, Algorithms and Computation in Mathematics, 7 (Springer, Berlin, 2000).

[Gal63] D. Gale, Neighborly and Cyclic Polytopes, Proceedings of Symposia in Pure Mathematics, VII (American Mathematical Society, Providence, RI, 1963), 225-232. MR 0152944.

[Imm95] N. Immerman, 'Descriptive complexity: a logician's approach to computation', Not. Amer. Math. Soc. 42(10) (1995), 1127-1133. MR 1350010.

[Isi19] M. Umut Isik, 'Complexity classes and completeness in algebraic geometry', Found. Comput. Math. 19(2) (2019), 245-258. MR 3937954.

[Joh02] P. T. Johnstone, Sketches of an Elephant: a Topos Theory Compendium, Oxford Logic Guides, 431 (The Clarendon Press, Oxford University Press, New York, 2002), MR 1953060.

[Kal88] E. Kaltofen, 'Greatest common divisors of polynomials given by straight-line programs', J. ACM (JACM) 35(1) (1988), 231-264.

[KR00] M. Kreuzer and L. Robbiano, Computational Commutative Algebra. 1 (Springer, Berlin, 2000), MR 1790326.

[LS88] J. Lambek and P. J. Scott, Introduction to Higher Order Categorical Logic, Cambridge Studies in Advanced Mathematics, 7 (Cambridge University Press, Cambridge, 1988), Reprint of the 1986 original. MR 939612. 
[MFK94] D. Mumford, J. Fogarty and F. Kirwan, Geometric Invariant Theory, 3rd edn, Ergebnisse der Mathematik und ihrer Grenzgebiete (2) [Results in Mathematics and Related Areas (2)], 34 (Springer, Berlin, 1994), MR 1304906.

[ML98] S. M. Lane, Categories for the Working Mathematician, 2nd edn, Graduate Texts in Mathematics, 5 (Springer, New York, 1998), MR 1712872.

[MLM94] S. M. Lane and I. Moerdijk, Sheaves in Geometry and Logic, Universitext (Springer, New York, 1994), A first introduction to topos theory, Corrected reprint of the 1992 edition. MR 1300636 MR 1300636.

[MO15] D. Mumford and T. Oda, Algebraic Geometry. II, Texts and Readings in Mathematics, 73 (Hindustan Book Agency, New Delhi, 2015), MR 3443857.

[Poi95] B. Poizat, Les petits cailloux, Nur al-Mantiq wal-Ma'rifah [Light of Logic and Knowledge], 3, Aléas, Lyon, 1995, Une approche modèle-théorique de l'algorithmie. [A model-theoretic approach to algorithms]. MR 1333892.

[Sim11] H. Simmons, An Introduction to Category Theory (Cambridge University Press, Cambridge, 2011), MR 2858226.

[Str73] V. Strassen, 'Vermeidung von divisionen', J. Reine Angew. Math. 264 (1973), 184-202.

[Val79a] L. G. Valiant, 'Completeness classes in algebra', in Proceedings of the Eleventh Annual ACM Symposium on Theory of Computing (ACM, New York, NY, USA, 1979), 249-261.

[Val79b] L. G. Valiant, 'The complexity of computing the permanent', Theoret. Comput. Sci. 8(2) (1979), 189-201.

[vzG87] J. von zur Gathen, 'Feasible arithmetic computations: Valiant's hypothesis', J. Symbolic. Comput. 4(2) (1987), 137-172.

[Yan15] N. S. Yanofsky, 'Computability and complexity of categorical structures', Preprint, 2015, CoRR arXiv:1507.05305. 